\title{
1 Mean-field thalamocortical modeling of longitudinal EEG 2 acquired during intensive meditation training
}

3
Manish Saggar ${ }^{1,2}$, Anthony P. Zanesco ${ }^{3,4}$, Brandon G. King ${ }^{3,4}$, David A. Bridwell ${ }^{5}$, Katherine A. MacLean ${ }^{6}$, Stephen R. Aichele ${ }^{3,4}$, Tonya L. Jacobs ${ }^{4}$, B. Alan Wallace ${ }^{7}$, Clifford D. Saron ${ }^{4,8}$, and Risto Miikkulainen ${ }^{2}$

${ }^{1}$ Department of Psychiatry and Behavioral Sciences, Stanford University, Stanford, CA, USA

${ }^{2}$ Department of Computer Science, University of Texas at Austin, TX, USA

${ }^{3}$ Department of Psychology, University of California, Davis, CA, USA

${ }^{4}$ Center for Mind and Brain, University of California, Davis, CA, USA

${ }^{5}$ Mind Research Network, Albuquerque, NM, USA

${ }^{6}$ Department of Psychiatry and Behavioral Sciences, Johns Hopkins University, Baltimore, MD, USA

${ }^{7}$ Santa Barbara Institute for Consciousness Studies, Santa Barbara, CA, USA

${ }^{8}$ The M.I.N.D. Institute, University of California, Davis, Sacramento, CA, USA

Running Title:

Modeling changes in cortical activity associated with meditation training

Number of words in Abstract: 311

Number of words in Introduction: 1905

Number of words in Methods: 2508

Number of words in Results: 1640

Number of words in Discussion: 3667

Number of Figures: 8

Number of Tables: 4

\section{Corresponding Author:}

Manish Saggar, Ph.D.

401 Quarry Road, MC 5795,

Department of Psychiatry and Behavioral Sciences, Stanford University, Stanford, CA, USA.

E-mail: saggar@stanford.edu 


\section{Abstract}

43 Meditation training has been shown to enhance attention and improve emotion regulation.

44 However, the brain processes associated with such training are poorly understood and a

45 computational modeling framework is lacking. Modeling approaches that can realistically

46 simulate neurophysiological data while conforming to basic anatomical and physiological

47 constraints can provide a unique opportunity to generate concrete and testable hypotheses

48 about the mechanisms supporting complex cognitive tasks such as meditation. Here we

49 applied the mean-field computational modeling approach using the scalp-recorded

50 electroencephalogram (EEG) collected at three assessment points from meditating

51 participants during two separate 3-month-long shamatha meditation retreats. We modeled

52 cortical, corticothalamic, and intrathalamic interactions to generate a simulation of EEG

53 signals recorded across the scalp. We also present two novel extensions to the mean-field

54 approach that allow for: (a) non-parametric analysis of changes in model parameter

55 values across all channels and assessments; and (b) examination of variation in modeled

56 thalamic reticular nucleus (TRN) connectivity over the retreat period. After successfully

57 fitting whole-brain EEG data across three assessment points within each retreat, two

58 model parameters were found to replicably change across both meditation retreats. First,

59 after training, we observed an increased temporal delay between modeled cortical and

60 thalamic cells. This increase provides a putative neural mechanism for a previously

61 observed reduction in individual alpha frequency in these same participants. Second, we

62 found decreased inhibitory connection strength between the TRN and secondary relay

63 nuclei (SRN) of the modeled thalamus after training. This reduction in inhibitory strength

64 was found to be associated with increased dynamical stability of the model. Altogether,

65 this paper presents the first computational approach, taking core aspects of physiology

66 and anatomy into account, to formally model brain processes associated with intensive

67 meditation training. The observed changes in model parameters inform theoretical

68 accounts of attention training through meditation, and may motivate future study on the

69 use of meditation in a variety of clinical populations.

71 Keywords

72 Computational Modeling, EEG, Meditation Training, Mean-field modeling, Alpha

73 Frequency, Beta-band Power 


\section{1. Introduction}

77 In traditional Buddhist thought, meditation refers to a process of familiarization (Tibetan

78 gom) with or cultivation (Sanskrit bhavana) of particular mental states and cognitive

79 capacities through the repeated observation, investigation, and recollection of mental

80 processes and events (Langri, 2009; Wallace, 2005). Meditation is thus conceptualized as

81 a form of mental training in which the practitioner engages in mental exercises in order

82 to develop beneficial psychological, cognitive, and motivational traits (Walsh \& Shapiro,

83 2006; Lutz, Slagter, Dunne, \& Davidson, 2008), and attempts to gain deeper insight into

84 their mental life. This conceptualization of meditation as a developmental process shares

85 considerable theoretical overlap with cognitive and neuroscientific theories of learning,

86 development, and neuroplasticity (Slagter et al., 2011). Contemporary psychological

87 accounts of meditation have therefore argued that it is possible to understand and

88 characterize the neurocognitive framework associated with meditation in terms of

89 established features of attention and cognitive control (e.g., Hölzel et al., 2011; Lutz et

90 al., 2008).

91 Among the practices utilized within various Buddhist traditions are a class of

92 attention-regulatory techniques designed to promote attentional stability and vividness,

93 traditionally termed shamatha (lit. calm abiding; Wallace, 2006; 2005). During shamatha

94 practice, practitioners voluntarily direct and maintain attention on an external or internal

95 object or domain of focus (e.g., sensations of breath), monitoring if attention is on the

96 intended target, and gently reorienting attention whenever it strays or becomes lax. A

97 number of studies have shown that training with shamatha and related focused-attention

98 (FA) meditation techniques is associated with enhanced cognitive control and improved 
99 attention regulation. For example, studies of intensive training in such meditation

100 techniques have been shown to improve attentional stability (Lutz et al., 2009; MacLean

101 et al., 2010) and alerting (Jha et al., 2007), sustained response inhibition (Sahdra et al.,

102 2011), efficiency in information processing (Slagter et al., 2007; van Vugt and Jha,

103 2011), and perceptual discrimination (MacLean et al., 2010). Moore and colleagues

104 reported that regular but non-intensive practice of FA-style meditation over 16 weeks can

105 lead to enhanced attentional processing and increased efficiency of resource allocation

106 during object recognition processes (Moore et al., 2012). Overall, these studies have

107 shown that regular practice of shamatha and related FA meditation may lead to

108 improvements in behavioral measures of attention and cognitive control.

109 Despite the sizeable amount of attention-related research on meditation training,

110 the brain processes associated with these behavioral improvements are not well

111 characterized. One avenue for investigating the neural correlates of meditation has been

112 to examine the patterns of brain activation that co-occur during the practice of specified

113 meditation techniques. These studies have typically relied upon measuring cortical

114 oscillatory activity (scalp-recorded EEG) and have more recently employed other

115 neuroimaging modalities such as functional Magnetic Resonance Imaging (Cahn \&

116 Polich, 2006). Although a number of studies have suggested that experienced meditation

117 practitioners show greater recruitment of attention-related brain networks during practice

118 of FA meditation (Brefczynski-Lewis et al., 2007; Hasenkamp \& Barsalou, 2012;

119 Hasenkamp et al., 2012; Saggar et al., 2012), there is no consensus on the extent or

120 specificity of neural activity associated with such practice. Investigation of the neural

121 processes recruited during FA practice is complicated by the difficulty of experimentally 
122 controlling participants' unobservable mental states, which cannot be corroborated

123 conclusively through external measurement. Thus, much of this research relies on

124 inferences regarding the engagement of specific cognitive processes drawn solely from

125 observed neural activity. Furthermore, relatively little is known about how intensive

126 training alters state-specific neural activity. Characterizing how meditation state-specific

127 neural activity may be affected through training (e.g., Saggar et al., 2012) will aid our

128 understanding of how the repeated recruitment of large-scale brain networks during

129 meditation practice may be related to the development of enduring psychological traits.

130 In our previous work (Saggar et al., 2012), we examined patterns of scalp-

131 recorded oscillatory activity (EEG) while participants engaged in six minutes of

132 mindfulness of breathing practice in which they focused on the tactile sensations of the

133 breath. Training and wait-list control group participants each underwent a three-month

134 intensive shamatha meditation retreat. Ongoing cortical oscillatory activity was assessed

135 using spectral analysis of dense-array EEG at three assessment points (pre-, mid-, and

136 post-retreat) across two separate training periods. Two robust changes in cortical activity

137 were replicated across the training interventions: 1) significant reductions in beta-band

138 power, bilaterally over anterior-central and posterior scalp regions, and 2) reductions in

139 state-related global individual alpha frequency (IAF; Klimesch, 1999). Training-related

140 changes in beta-band power were interpreted as indicating increased cortical activation of

141 sensory- and attention-related brain networks recruited during voluntary focus on the

142 tactile sensations of the breath. In the context of these changes in spectral power,

143 reductions in IAF were interpreted as suggesting that participants' capacity to focus

144 attention on the breath was less effortful following training. These findings provide 
145 evidence of longitudinal changes in meditation state-related brain oscillatory activity

146 during mindfulness of breathing that may potentially support long-term improvements in

147 attention regulation.

148 In studies such as Saggar et al., 2012, the ability to draw clear conclusions about

149 psychological processes from presumed meditation state-related brain activity is limited

150 by a number of methodological and inferential constraints. During meditation practice,

151 meditation practitioners typically engage in covert or internalized tasks for which there

152 are no obvious external markers of performance or compliance. Although researchers

153 often provide specific instructions to practitioners to engage in particular meditation

154 techniques, there is no direct way to confirm that participants are following instructions

155 similarly. Brain activity observed during meditation may also reflect cognitive states not

156 directly related to implementing the techniques themselves (e.g., mind wandering).

157 Inferences regarding the role of observed brain activity as reflecting state-specific

158 cognitive processes must rely on references to patterns of activity identified in other task

159 domains. Potential avenues for resolving these ambiguities include pairing induced state-

160 related brain activity with practitioners' first-person introspective reports (i.e.,

161 neurophenomenology; Desbordes \& Negi, 2013; Lutz \& Thompson, 2003), and

162 correlational approaches that relate measures of meditation state-related brain activity to

163 behavioral performance on tasks presumed to share cognitive mechanisms with specified

164 techniques. Another approach involves utilizing computational models of state-related

165 activity to better characterize presumed mechanisms of neural activation from the

166 recorded EEG itself. Such models can be used to generate targeted hypotheses regarding

167 the cortico-cortical and subcortico-cortical dynamics associated with practice and training 
168 (Kerr et al., 2013). Here, we utilize the later approach of computational modeling to

169 formally characterize longitudinal changes in cortical activity associated with intensive

170 focused-attention meditation training (Saggar et al., 2012). Computational models

171 provide a mathematical approach that allows for simulating complex phenomena for

172 which closed-form analytical solutions do not exist and for generating concrete novel

173 hypotheses for future research.

174 Effective computational models are based on realistic biophysical and anatomical

175 constraints involving conceptual understanding of the processes involved. The cognitive

176 model of attention proposed by Posner and Peterson (1990) articulates a number of

177 attentional component processes that are relevant to conceptions of shamatha and related

178 FA meditation practice, such as attentional alerting, orienting, and monitoring.

179 Neuroimaging studies investigating this cognitive model (Corbetta \& Shulman, 2002;

180 Fan et al., 2005) have highlighted the role of fronto-parietal cortical networks and

181 thalamic sub-cortical areas in attention regulation (Kastner et al., 2012) and cognitive

182 control (Dosenbach et al., 2008). Additionally, these findings have been corroborated by

183 neuroimaging studies of large-scale cognitive-control networks (Bressler \& Menon,

184 2010; Menon, 2011). Based on these studies, we propose to model the neurophysiological

185 mechanisms associated with the cognitive processes engaged during FA meditation by

186 incorporating cortical and thalamic components involved in attention regulation and by

187 investigating the utility of a biophysical model of corticothalamocortical loops to account

188 for observed longitudinal changes in scalp-recorded EEG.

189 There are a wide range of approaches available for the computational modeling of

190 EEG data, including purely phenomenological approaches (Isaksson et al., 1981; Wright 
191 et al., 1990), mean-field modeling (i.e., Freeman, 1987; 1972; Jirsa et al., 2010; Lopes da

192 Silva et al., 1974; Nunez, 1974a; 1974b; Robinson et al., 2001b; Wilson \& Cowan, 1973,

193 1972), and detailed neural networks (Lagerlund \& Sharbrough, 1989; Lumer et al., 1997;

194 Reimann et al., 2013; Traub et al., 1997; Wilson and Bower, 1992). Whereas

195 phenomenological approaches simulate data without incorporating anatomical

196 information, mean-field modeling allows for simulation at the level of neural populations

197 while incorporating some anatomical information. Discounting volume conduction, each

198 EEG sensor ( $\sim 5 \mathrm{~mm}$ in diameter) represents the aggregate synaptic activity of a

199 population of hundreds of thousands of neurons (Pizzagalli, 2007). The complexities of

200 population dynamics, resulting from the complexity of cortical micro-circuitry creates a

201 challenge for detailed modeling. However, an approximation of mean population activity

202 may be modeled for each sensor using mean-field modeling, while keeping intact desired

203 assumptions regarding overall corticothalamic anatomical and physiological constraints.

204 We used the Robinson et al. (2001b) Mean-Field Model (henceforth referred to as

205 R-MFM), in which EEG spectra at the scalp are simulated using postulated

206 corticocortical, corticothalamic, and intrathalamic loops. The R-MFM was chosen

207 because scalp-recorded EEG data are generated primarily by the summation of excitatory

208 and inhibitory post-synaptic potentials in the pyramidal cells of the cortex (Pizzagalli,

209 2007; Speckmann \& Altrup, 1993), and because oscillations in subcortical areas,

210 especially the thalamus, and oscillations due to corticothalamocortical interactions are

211 considered to be major contributors to the generation of cortical alpha and beta rhythms

212 (Robinson et al., 2002; Steriade, 2005; Steriade et al., 1993). In humans, studies

213 combining EEG measures with positron emission tomography and fMRI have provided 
214 direct evidence for a relation between glucose metabolic activity in the thalamus and

215 scalp-recorded EEG alpha power (Goldman et al., 2002; Larson et al., 1998;

216 Schreckenberger et al., 2004). In a recent review, using a neural network model of a

217 single cortical column in the primary somatosensory cortex (SI) (Jones et al., 2007,

218 2009), Kerr et al. (2013) hypothesized that interactions between thalamic regions and SI

219 may facilitate attentional modulation of alpha rhythms $(7-14 \mathrm{~Hz})$ during FA meditation

220 practice. Further, research on long-term meditators has also invoked the role of thalamic

221 nuclei (esp. the reticular nucleus) in regulating and sustaining attention on the object of

222 meditative focus (Austin, 2013; Guglietti et al., 2012; Newberg \& Iversen, 2003). Thus,

223 by modeling interactions between corticocortical, corticothalamic and intrathalamic cells

224 in meditation-state related EEG data, our computational approach can provide a

225 framework for generating targeted hypotheses, which can motivate empirical verification

226 using multimodal non-invasive approaches in future research.

227 In the present investigation, we first used R-MFM to simulate whole-brain EEG

228 data obtained while participants engaged in six minutes of breath-focused FA meditation

229 practice. Next, using standard model-fitting procedures, the R-MFM parameters were

230 refined for each participant in order to fit the simulated data to the observed EEG spectra

231 collected at three assessment points over the 3-month training period. Finally, using

232 inverse computational modeling, we explored longitudinal changes in model parameters

233 to examine the effects of intensive meditation training on changes in corticocortical,

234 corticothalamic, and intrathalamic parameters.

235 We also present two novel extensions to the R-MFM. First, using nonparametric

236 statistical testing, we analyze longitudinal changes in model parameters across the whole- 
237 brain, rather than for single channels, as was done in previous applications of R-MFM;

238 (Robinson et al., 2003). This approach allows for analysis of changes in whole-brain EEG

239 topography associated with intensive meditation training. Second, we explore how

240 simulated connectivity patterns in the TRN may change over time. This extended model

241 will henceforth be referred to as ER-MFM. Although these proposed extensions were

242 developed to analyze meditation data, they can be applied to model longitudinal changes

243 in corticothalamic architecture and dynamics within any EEG or MEG dataset.

\section{2. Methods}

246 2.1 Study Design:

247 Two three-month long residential meditation retreats were held at a scenic meditation

248 center (Shambhala Mountain Center) in Red Feather Lakes, CO. Participants lived and

249 practiced meditation onsite for the duration of training. Two separate groups of 30

250 participants were tested during the initial 3-month retreat: an in-residence retreat group

251 undergoing training (RG1) and a matched wait-list control group (CG) that was flown to

252 the retreat center for each assessment. The $\mathrm{CG}$ did not receive training during this period,

253 but was tested using procedures identical to RG1. In the second retreat, the same CG

254 participants underwent training that was formally identical to the training of RG1

255 participants during the initial retreat. Thus, we analyzed three datasets from two groups

256 of participants: RG1 and CG during the initial retreat, and CG as training participants

257 during the second retreat (subsequently referred to as RG2). The institutional review

258 board of the University of California, Davis, approved all study procedures. 
261 Data from 22 individuals in each participant group (initial retreat and wait-list control)

262 were included in all analyses. Data for the remaining participants were removed due to

263 poor signal quality and technical issues (see Saggar et al, 2012). Initial retreat and wait-

264 list control participants included in the final sample did not differ (all $p$ s $>.05$ ) in age

265 (RG1: $M=49.5$ years, $S D=13.5$, CG: $M=44.2$ years, $S D=15.8$ ), gender (RG1: 12

266 female, CG: 11 female), or estimated lifetime meditation experience (RG1: $M=2855.6$

267 hours, $S D=2994.1$, CG: $M=2272.7$ hours, $S D=2326.3$ ).

2692.3 Training:

270 Dr. B. Alan Wallace, an established Buddhist teacher, contemplative, and scholar, served 271 as the meditation instructor during both retreats. Meditation training was comprised of 272 two general classes of techniques from the Buddhist contemplative tradition: shamatha 273 techniques involving directing and sustaining attention on a chosen object and ancillary 274 techniques involving the generating benevolent aspirations for the well-being of oneself 275 and others (Sahdra et al., 2011; Wallace, 2006). Shamatha techniques included 276 mindfulness of breathing, in which attention is directed toward the breath; observing

277 mental events, in which attention is directed toward the whole field of mental experience 278 (thoughts, images, sensations); and observing the nature of consciousness, in which 279 attention rests in the clear and cognizant experience of being aware. These shamatha 280 practices are defined as such because they each aim to cultivate stable, clear, and 281 effortless, single-pointed concentration (Wallace, 2006). Although these practices 282 incorporate features commonly ascribed to focused-attention techniques, they may 
283 additionally share features of other classes of techniques, such as open-monitoring (OM;

284 Lutz et al., 2008). Beneficial aspirations included practices that aim to cultivate loving-

285 kindness, compassion, empathic joy, and equanimity (Wallace, 2006). Participants in

$286 \mathrm{RG} 1(\mathrm{~N}=22)$ spent an average of 5.7 hours per day $(S D=1.5)$ practicing shamatha

287 techniques (mindfulness of breathing $M=2.10$ hours, $S D=2.16$; observing mental events

$288 M=1.74$ hours, $S D=2.22$; and observing nature of consciousness $M=1.86$ hours, $S D=$

289 2.11), and participants in $\mathrm{RG} 2(\mathrm{~N}=22)$ spent an average of 5.4 hours per day $(S D=1.5)$

290 on shamatha techniques (mindfulness of breathing $M=3.16$ hours, $S D=1.84$; observing

291 mental events $M=1.00$ hours, $S D=1.23$; and observing nature of consciousness $M=$

2921.19 hours, $S D=1.12)$. The retreat groups $(\mathrm{RG} 1, \mathrm{RG} 2)$ did not differ on the amount of

293 time spent practicing solitary shamatha meditation $(t(42)=0.49, p=.63)$. Participants

294 met twice daily for group meditation practice and discussion guided by Dr. Wallace.

295 Participants also met one-on-one with Dr. Wallace on a weekly basis for advice,

296 clarification, and guidance.

297

2982.4 EEG data collection and preprocessing

299 Dense-array scalp EEG was recorded from 88 locations (equidistant montage,

300 www.easycap.de) using a Biosemi Active2 system (www.biosemi.com) with 24-bit

301 resolution sampled at $2048 \mathrm{~Hz}$, while participants engaged in a 12-min period of silent,

302 eyes-closed mindfulness of breathing FA meditation. Participants were assessed at the

303 beginning (T1), middle (T2), and end (T3) of each three-month retreat. During each

304 assessment, the meditation began with approximately $50 \mathrm{~s}$ of audio instructions (provided

305 below), recorded by Dr. Wallace: 
"During the next 12 minutes, engage in the practice of mindfulness of breathing, focusing your attention on the tactile sensations at the apertures of your nostrils or just above your upper lip. With each inhalation arouse your attention and focus clearly on these tactile sensations. With each out-breath continue to maintain your attention upon the tactile sensations, while relaxing your body and mind, releasing any involuntary thoughts that may arise. So in this way maintain an ongoing flow of mindfulness, arousing with each in-breath, relaxing with each out-breath.”

314 A recorded sound ("chime") signaled the end of the 12-minute meditation period.

315 Continuous EEG was recorded over this entire period. However, due to an error in data 316 acquisition at T1, only the first six minutes of data were recorded for some subjects. To 317 allow for comparisons across all time points and datasets, we restricted analyses to the 318 first six minutes of meditation across all three assessments in both retreats.

319 The 88-channel EEG data were band-pass filtered offline between 0.1 and 200

320 Hz. Second-order blind source identification (Belouchrani et al., 1993) was used to derive

321 and separate non-neural signal contaminants from the ongoing electrical brain activity.

322 Sources of putative non-neural origin were identified using a novel semi-automatic

323 artifact removal tool (SMART, available at http://stanford.edu/ saggar/Software.html).

324 Extensive details regarding artifact identification and removal procedures can be found in

325 Saggar et al. (2012). Special attention was given to remove sources due to muscular 326 artifacts (aka electromyogram or EMG). Such sources were identified using low auto

327 correlation values, broadband power across all frequencies and localized topographical

328 locations (Saggar et al. 2012). After artifact removal, the 88-channel EEG data were 
329 reconstructed and transformed into a standard 81-channel montage (international 10-10

330 system), using spherical spline interpolation (Perrin et al., 1989). Interpolations were

331 accomplished using Brain Electrical Source Analysis software (BESA 5.2; www.besa.de)

332 with a smoothing parameter value $(\lambda)$ of $2 \times 10^{-6}$. This transformation ensured that

333 channel locations were standardized and that the number of channels remained consistent

334 across participants. Eight channels (AF9, Fp1, Fpz, Fp2, Nz, AF10, CB1, CB2) from the

335 81-channel montage were excluded because information from the nearest corresponding

336 electrode sites were not fully available in the original montage, yielding a final 73-

337 channel montage for the reconstructed EEG. These reconstructed data were then

338 transformed to a reference-free estimation of scalp current density (CSD; Kayser \&

339 Tenke, 2006).

340

3412.5 Experimental EEG Measures: Power spectrum and individual alpha frequency

342 estimation

343 The six minutes of reconstructed, artifact-free continuous EEG data were divided into

344 two-second segments with $75 \%$ overlap. Power spectra were then calculated for each of

345 these segments using the multi-tapered power spectral density estimation method (Mitra

346 \& Pesaran, 1999; Oostenveld et al., 2011) using in-house scripts implemented in

347 MATLAB (MATLAB, 2010). Multi-tapered estimation improves power spectra

348 estimation by obtaining multiple estimates from each sample.

349 Individual alpha frequency (IAF) was estimated using the center of gravity

350 method for the frequency range of $7 \mathrm{~Hz}\left(\mathrm{f}_{1}\right)$ to $14 \mathrm{~Hz}\left(\mathrm{f}_{2}\right)$ (Klimesch, 1999), 


$$
\alpha_{\mathrm{IAF}}=\frac{\sum_{\mathrm{i}=\mathrm{f}_{1}}^{\mathrm{f}_{2}}\left(\mathrm{a}\left(\mathrm{f}_{\mathrm{i}}\right) \times \mathrm{f}_{\mathrm{i}}\right)}{\sum_{\mathrm{i}=\mathrm{f}_{1}}^{\mathrm{f}_{2}}\left(\mathrm{a}\left(\mathrm{f}_{\mathrm{i}}\right)\right)},
$$

351 where power-spectral estimates at frequency $f_{i}$ are denoted by $a\left(f_{i}\right)$. The $\alpha_{\text {IAF }}$ values were

352 calculated for each channel and were averaged across all channels to obtain a single IAF

353 value per participant.

354

$355 \quad 2.6$ Model Architecture

356 The Robinson et al. (2001) mean-field modeling approach employs

357 corticothalamic architecture and dynamics to simulate scalp EEG, and is based on earlier

358 works on continuum modeling of EEG (Freeman, 1975; Liley \& Wright, 1994; Lopes da

359 Silva et al., 1974; Nunez, 1974a; Rennie et al., 1999; Robinson et al., 1997; 1998; Wilson

360 \& Cowan, 1973). This approach incorporates basic neurophysiological principles and

361 structures, including transmission delays in axonal propagation, excitatory and inhibitory

362 neural populations, and length- or range-dependent connections between cortical and

363 subcortical populations (an overview is presented in Figure 1 and a description of

364 physiological limits is listed in Table 1). The R-MFM has been successfully used to

365 reproduce various temporal and spectral properties of EEG data, such as evoked

366 potentials (Kerr et al., 2008; 2010), seizure dynamics (Breakspear et al., 2006; Robinson

367 et al., 2002), scalp-recorded EEG power spectra (Rennie et al., 2002; Robinson et al.,

368 2001b; Rowe et al., 2004a), inter-channel coherence and correlation (Robinson et al.,

369 2003), and changes due to aging in healthy adults (Kerr et al., 2010; 2011; van Albada et

370 al., 2010). 
373 First, all 73 EEG channels were independently modeled using the R-MFM. This approach

374 assumes spatial uniformity and local independence in model parameters (i.e., a change in

375 a parameter value at a distant channel will not affect the spectrum of the channel under

376 observation). Thus, while modeling each channel, the values of parameters are chosen

377 such that the modeled spectrum for each channel matches the experimental EEG

378 spectrum. This approach is known as the local effective value (LEV) model (O'Connor

379 and Robinson, 2004) and, although a first approximation, is both analytically tractable

380 and computationally light (O'Connor and Robinson, 2004). For eyes-closed states, it has

381 been shown that the local independence assumption of the LEV model is maintained

382 within constraints of the model, except for low frequencies (i.e., $<2 \mathrm{~Hz}$ ) and at alpha

383 frequency. However, this non-independence at alpha frequency is much smaller in

384 magnitude than analogous effects at lower frequencies (O’Connor and Robinson, 2004).

385 Although care was taken to remove artifactual sources of noise (especially

386 electromyographic activity (EMG) due to scalp muscle tension) during preprocessing and

387 post-SOBI reconstruction of EEG data, a parameter for remaining EMG artifact was

388 included in the estimation of power spectrum for each channel, as follows,

$$
P_{\text {est }}(f)=P_{E E G}(f)+P_{E M G}(f) \text {. }
$$

389 No additional smoothing was performed on the experimentally recorded spectrum. The

$390 \quad \chi^{2}$ error between the estimated spectrum $\left(\mathrm{P}_{\mathrm{est}}(\mathrm{f})\right)$ and the experimentally obtained

391 spectrum $\left(\mathrm{P}_{\exp }(\mathrm{f})\right)$ was reduced using the trust-region-reflective constrained optimization

392 algorithm (Coleman \& Li, 1993), implemented in MATLAB, in which $\chi^{2}$ is calculated 393 for each site as 


$$
\chi^{2}=\sum_{i=1}^{N}\left[\log \left(P_{\exp }\left(f_{i}\right)\right)-\log \left(P_{\text {est }}\left(f_{i}\right)\right)\right]^{2}
$$

394 The range for parameter values was bounded using the physiology-based limits presented 395 in Table 1.

$397 \quad 2.8$ Model Extension 1: Longitudinal Analysis

398 To model longitudinal changes in EEG data associated with meditation training, the R-

399 MFM was extended to investigate changes in parameter values across assessment points

400 (T1, T2, and T3) for all 73 channels separately for both retreats. In this extended

401 approach (ER-MFM), spatiotemporal changes in model parameters were examined using

402 nonparametric cluster-based permutation testing (Maris \& Oostenveld, 2007) using

403 FieldTrip (Oostenveld et al., 2011), an open-source toolbox implemented in MATLAB.

404 This approach has been used previously for analyzing changes in power spectra and

405 coherence metrics in MEG and EEG data (Maris \& Oostenveld, 2007; Maris et al., 2007).

406 Our procedure for investigating changes in model parameters is shown schematically in

407 Figure 2 and is detailed in Algorithm 1.

408

\section{Algorithm 1: Group-level nonparametric statistical test for each R-MFM parameter \\ $\mathrm{p}_{\mathrm{i}}$}

Null Hypothesis $\left(H_{0}\right): \mathrm{p}_{\mathrm{i}}$ does not differ across the three assessments points

1. Collect $\mathbf{p}_{\mathrm{i}}$ for all 73 -channels across all subjects and assessments into a single super-set.

2. Randomly partition the super-set into three equal sets of subjects. 
3. Calculate the cluster-based test-statistic as follows:

a. For every channel, compare the parameter value $\mathrm{p}_{\mathrm{i}}$ across the three assessment-points, using the corresponding F-test statistic.

b. Select all channels with F-values larger than a given threshold.

c. Cluster the selected channels in connected sets on the basis of spatial adjacency.

d. Calculate the cluster-level F-statistic by taking a sum over all F-values within a cluster.

e. Output the largest of the cluster-level statistics.

4. Repeat Steps 2 and 3 a large number of times (e.g., 10,000) and construct a histogram of the test statistics.

5. Using the observed test-statistic and the histogram from Step 4, estimate the proportion of random partitions that resulted in a larger test-statistic than the observed test statistic. This proportion is the p-value.

6. If the $\mathrm{p}$-value is less than the designated critical alpha-level ( $p=0.05$, corrected for False Discovery Rate), then conclude that the null hypothesis $\mathrm{H}_{0}$ is rejected and that the parameter $\mathbf{p}_{\mathbf{i}}$ is significantly different across assessment points.

\section{$410 \quad 2.9$ Model Extension 2: TRN connectivity}

411 The original R-MFM, as described above, incorporates interactions between cortex,

412 reticular nucleus (TRN), and the specific relay nucleus (SRN) of the thalamus. However,

413 it does not account for within-TRN interactions. One potential approach for modeling

414 lateral connectivity in the TRN is to simulate and fit EEG power spectra concurrently 
415 across all EEG channels, an approach referred to as non-uniform R-MFM (O'Connor \&

416 Robinson, 2004; Robinson et al., 2003). In this non-uniform approach, interactions

417 between the modeled TRN cells can be retrieved after concurrent data simulation and

418 fitting. The difficulty, however, is that in addition to fitting the 10 usual parameters per

419 channel (see Table 1), simulating and fitting the coupled connectivity between 73 TRN

420 cells (due to 73-channel EEG data) would come at the cost of increased computational

421 resources and putative loss of analytical tractability in some cases (O'Connor \&

422 Robinson, 2004).

423 To address this challenge without resorting to a non-uniform modeling approach,

424 we developed a novel procedure to model TRN's lateral connectivity and to explore

425 changes in such connectivity associated with meditation training (Figure 3). After model

426 fitting of experimental spectra for all electrodes, white noise was injected into the

427 modeled cortical cells (Figure 3C). The activity due to the injected noise in the modeled

428 TRN cells was extracted using linear algebra based on the original time domain R-MFM

429 equations:

$$
\phi_{\mathrm{r}}(\mathrm{t})=\frac{\phi_{\mathrm{e}}\left(\mathrm{t}-\frac{\mathrm{t}_{\mathrm{o}}}{2}\right)\left[\mathrm{G}_{\mathrm{esre}}+\mathrm{G}_{\mathrm{ese}} \mathrm{G}_{\mathrm{srs}}\right]+\phi_{\mathrm{n}}(\mathrm{t})\left[\mathrm{G}_{\mathrm{es}} \mathrm{G}_{\mathrm{sn}} \mathrm{G}_{\mathrm{srs}}\right]}{\left(\mathrm{G}_{\mathrm{es}} \mathrm{G}_{\mathrm{sr}}\right)\left(1-\mathrm{G}_{\mathrm{srs}}\right)},
$$

430 where $G_{s n}=1, G_{e s}=\sqrt{ } G_{e s e}$, and $G_{s r}=\sqrt{ }\left|G_{\text {srs }}\right|$. In order to analyze TRN connectivity,

431 correlations between the extracted signals from TRN cells were computed (a high

432 correlation between two TRN cells would indicate higher connectivity). For robust

433 estimation, many iterations $(n=100)$ of noise injection and correlation matrix estimation

434 were performed for each cell. The correlation matrix was then averaged over all trials and

$435 \mathrm{k}$-means clustering was performed on the resulting matrix. The value of $\mathrm{k}$ was chosen

436 based on the visual inspection of the dendrogram generated by hierarchical clustering 
437 (MATLAB, 2010). Longitudinal changes in the structure and the connectivity of these

438 clusters were evaluated using non-parametric statistical testing (Algorithm 1).

\section{$440 \quad 2.10$ Stability Analysis.}

441 Using linear stability analysis, Robinson et al. (2002) suggested that low-frequency

442 instabilities of the brain can be modeled in a reduced three-dimensional parameter space

$443<x y z>$, where, $x=\frac{G_{e e}}{1-G_{e i}}, y=\frac{G_{e s e}+G_{e s r e}}{\left(1-G_{s r s}\right)\left(1-G_{e i}\right)}$, and $z=-\frac{G_{s r s} \alpha \beta}{(\alpha+\beta)^{2}}$. These parameters represent

444 cortical (x), corticothalamic (y), and intrathalamic stability (z), respectively. In their

445 procedure, Robinson et al. (2002) defined the stability zone for the modeled brain as the

$446<x y z>$ space under a three-dimensional surface defined by parameter values that induce

447 instabilities in different frequency bands and features of the EEG (alpha, theta, and

448 spindles). Thus, various brain states can be mapped onto this $<\mathrm{xyz}>$ space (see Figure 3

449 in Robinson et al., 2002).

450 To explore how observed longitudinal changes in model parameters may alter the

451 overall stability of modeled brain dynamics, fitted model parameters from the three

452 assessment points were mapped to the reduced $<x y z>$ space separately for each retreat.

453 As in the case of other model parameters, non-parametric statistical testing (Algorithm 1)

454 was used to longitudinally analyze changes in the reduced-set of stability parameters

$455(\mathrm{x}, \mathrm{y}$, and $\mathrm{z})$.

456

457 3. Results

458 All reported post-hoc Wilcoxon tests are Bonferonni-protected and $p$-values are corrected 459 when indicated. 


\section{$461 \quad 3.1$ Data fitting}

462 Using the LEV approach, experimental EEG power spectra were fitted independently for

463 all electrodes (73 channels), separately for each participant and assessment point. As

464 shown in Figure 4, the model was successful in recreating the recorded EEG spectra (see

465 Table 2 for goodness of fit results), while keeping the parameters within the

466 physiologically plausible range (Tables 3 and 4). To verify that the aggregate model

467 fitting procedure was unbiased across assessments, a non-parametric false-discovery-rate

468 (FDR)-based procedure was used to find differences in the sum-squared errors in fit for

469 each channel across the three assessments within each group of participants. Dependent

470 sample $F$-tests were used (with a Bonferroni-corrected alpha level of $p<0.05$ ) to test for

471 differences across assessments. No differences in fit accuracy were found across

472 assessments in any group, demonstrating that the model fitting was generally unbiased.

$474 \quad 3.2$ Longitudinal analysis of model parameters

475 As expected, no changes in any of the 10 model parameters were found across

476 assessments for the CG. Changes in two out of 10 model parameters were found to

477 replicate across retreats: intrathalamic gain $\left(\mathrm{G}_{\mathrm{srs}}\right)$ and cortico-thalamic delay $\left(\mathrm{t}_{0}\right)$. In RG1,

478 a decrease in intrathalamic gain was found in the right lateral parieto-occipital cluster of

479 modeled channels ( $p=0.01$, FDR corrected; Figure 5). Bonferroni-corrected (.05/3) post-

480 hoc Wilcoxon tests revealed a significant reduction at T2 $(p<0.001)$ and T3 $(p=0.007)$,

481 as compared to T1. No differences were found between T2 and T3. In RG2, a similar

482 pattern of decrease in intrathalamic gain was found in a right-parietal cluster of modeled 
483 channels ( $p=0.04$; Figure 5). Post-hoc Wilcoxon tests revealed a reduction in

484 intrathalamic gain at T2 $(p=0.014)$ and T3 $(p=0.001)$, as compared to T1. Again, no

485 difference was found between T2 and T3.

486 The cortico-thalamic delay parameter increased with meditation training (Figure

487 6). This effect was evident in both retreat groups. In RG1, an increase in the delay

488 parameter was found at bilateral parietal-occipital model locations $(p=0.003)$. Wilcoxon

489 tests revealed a significant increase at T2 $(p<0.001)$ and T3 $(p=0.007)$ when compared

490 to T1 and no change between T2 and T3. Similarly, in RG2, an increase was found at

491 right parietal-occipital modeled locations $(p=0.01)$. Wilcoxon tests revealed an increase

492 in delay parameter value at T2 $(p=0.001)$ and T3 $(p=0.001)$ when compared with T1,

493 but no change was found between T2 and T3.

494

4953.3 Stability analysis

496 To examine changes in stability of the modeled EEG dynamics in a reduced three-

497 dimensional parameter space, the cortical (x), corticithalamic (y), and intrathalamic (z)

498 stability parameters were analyzed separately. Only the intrathalamic stability parameter

$499 \mathrm{z}$ was found to change across assessments for both retreat groups (RG1: $p$ (FDR

500 corrected $)=0.011$ and RG2: $p=0.033$; Figure 7). The cluster locations found for $z$ were

501 identical to those found for $\mathrm{G}_{\text {srs }}$. This is because the $\mathrm{z}$ parameter, by definition, is directly

502 proportional to $G_{\text {srs }}$, which changed significantly as indicated in section 3.2. No cluster

503 was found for the CG. Therefore, for the purpose of analysis, clusters found for RG1

504 participants were used to extract data for post-hoc comparisons of CG participants within

505 Retreat 1, while clusters found for RG2 were used to extract CG data within Retreat 2. 
506 Thus, for Retreat 1 control data, CG cluster electrodes were not based on the clusters

507 found for these individuals themselves when in their own retreat. A Wilcoxon test for the

508 RG1 model cluster revealed a significant decrease in $\mathrm{z}$ at T2 $(p=0.002)$ and at T3 $(p=$

509 0.001), as compared to T1. As expected, there were no changes in z parameter values for

510 the corresponding cluster extracted from the modeled CG data. A Wilcoxon test for this

511 cluster in the RG2 model revealed a significant decrease in the z parameter at T2 ( $p=$

$5120.014)$ and T3 $(p=0.001)$, when compared to T1. Again, there was no change in $\mathrm{z}$ values

513 extracted from CG for the same cluster.

514

5153.4 TRN connectivity analysis

516 We used the second model extension (see Methods 2.9) to examine longitudinal changes

517 in intra-TRN connectivity. We took a three-part approach to this analysis. First, to

518 qualitatively examine the overall pattern of intra-TRN connectivity, we used k-means

519 clustering to identify two groups of TRN cells characterized by high within-cluster

520 correlations. To visualize the topography of these k-means clusters, we utilized the

521 topographical organization of the experimentally recorded EEG, where each of the

522 channels is an independently modeled instance of R-MFM (see example in Figure 8a).

523 Within the TRN layer, each cell in the anterior cluster was assigned +1 and each cell in

524 the posterior cluster was assigned -1. To create average "scalp" cluster topographies, we

525 averaged these +/-1 assignments across participants within each dataset and assessment.

526 Overall, we observed an anterior to posterior segregation of within-TRN layer

527 connectivity that is visualized at the group-level in Figure 8b. 
529 pattern. We used nonparametric cluster-based permutation testing on the topographies

530 created from anterior/posterior assignments based on inter-cell correlation values. No

531 significant changes were found across assessments for any group, indicating that this

532 feature of spatial segregation is stable with respect to shamatha and related FA-

533 meditation training.

534 Finally, to examine longitudinal changes in connectivity (i.e., mean correlation

535 values) both within and between the anterior and posterior clusters, we performed a

536 related-samples Friedman's Analysis of Variance by Ranks Tests (a non-parametric

537 alternative to parametric one-way ANOVA with repeated measures (Friedman, 1937))

538 separately for the three participant datasets (RG1, CG, and RG2). For each dataset, we

539 examined the null hypothesis that the distributions of within- and between-cluster

540 connectivity did not differ across time points. Out of the nine tests (i.e., 3 datasets x 3

541 connectivity tests ( 2 within-cluster and 1 between-cluster)), only two rejected the null

542 hypothesis (FDR-corrected $\mathrm{p}<0.05$ ), such that in both retreat groups (RG1 and RG2)

543 between-cluster connectivity was significantly different across time points (FDR-

544 corrected $p=0.0495$ for each dataset). In RG1, the post-hoc Wilcoxon tests revealed a

545 significant increase in between-cluster connectivity at T3 as compared to T1 $(p=0.046)$,

546 and no significant difference was found between $\mathrm{T} 1$ and $\mathrm{T} 2$ or between $\mathrm{T} 2$ and $\mathrm{T} 3$

547 (Figure 8c). In RG2, however, a significant increase in between-cluster connectivity was

548 observed at T2 $(p=0.010)$ compared with T1, (Figure 8c) and no differences were found

549 between $\mathrm{T} 1$ and $\mathrm{T} 3$ or between $\mathrm{T} 2$ and $\mathrm{T} 3$ (Figure $8 \mathrm{c}$ ). 
552 A series of regressions were used to examine relations between changes in model-derived

553 parameters and previously reported longitudinal changes in beta-band power and alpha-

554 frequency in this same dataset (Saggar et al., 2012). Because patterns of change in model

555 parameters and observed oscillatory activity were similar across retreats, RG1 and RG2

556 datasets were combined for the regression analyses.

557

558 3.5.1 Relating changes in model parameters to change in beta-band power

559 Neither intrathalamic gain nor corticothalamic delay at T1 were significant predictors of

560 beta-band power at $\mathrm{T} 1\left(\mathrm{R}^{2}=0.01, \mathrm{~F}(1,42)=0.028, \mathrm{p}=0.867\right.$ and $\mathrm{R}^{2}=0.013, \mathrm{~F}(1,42)=$

$5610.565, \mathrm{p}=0.456$, respectively).

562 We examined whether longitudinal changes in these two model parameters might

563 predict reductions in observed beta-band power. In the first multiple regression, beta-

564 band power and intrathalamic gain at $\mathrm{T} 1$ were included as predictors of beta-band power

565 at $\mathrm{T} 3$ to account for baseline levels in these predictors prior to training. These predictors

566 explained a significant amount of variance in $\mathrm{T} 3$ beta-band power $\left(\mathrm{R}^{2}=0.80, \mathrm{~F}(2,41)=\right.$

$56784.22, \mathrm{p}<0.001)$. In the second step, the addition of the intrathalamic gain parameter

568 value at T3 did not significantly add to the explained model variance $\left(\Delta R^{2}=0.001\right.$,

$569 \Delta \mathrm{F}(1,40)=0.21, \mathrm{p}=0.65)$.

570 In a second multiple regression, we used an identical analytic strategy to test the

571 predictive effects of corticothalamic delay on observed beta-band power. Again, beta-

572 band power at $\mathrm{T} 3$ served as the dependent variable and beta-band power and

573 corticothalamic delay at T1 were included as initial predictors. These predictors 
574 explained a significant amount of variance in T3 beta-band power $\left(\mathrm{R}^{2}=0.80, \mathrm{~F}(2,41)=\right.$

$57583.35, \mathrm{p}<0.001)$. Similar to the first hierarchical regression, the addition of T3

576 corticothalamic delay value did not significantly add to the explained model variance

$577\left(\Delta \mathrm{R}^{2}=0.002, \Delta \mathrm{F}(1,40)=0.45, \mathrm{p}=0.51\right)$. Altogether, collapsed across the retreats,

578 changes in observed beta-band power were not predicted by longitudinal changes in

579 either the model-predicted intrathalamic gain or corticothalamic delay parameters.

580

581 3.5.2 Relating changes in model parameters to change in individual alpha frequency

582 Intrathalamic gain marginally predicted IAF at $\mathrm{T} 1\left(\mathrm{R}^{2}=0.08, \mathrm{~F}(1,42)=3.62, p=0.064\right)$,

583 while corticothalamic delay at $\mathrm{T} 1$ significantly predicted IAF at $\mathrm{T} 1\left(\mathrm{R}^{2}=0.50, \mathrm{~F}(1,42)=\right.$ $58442.67, \mathrm{p}<0.001)$.

585 We next examined whether longitudinal changes in the two model parameters 586 could explain the longitudinal reduction in IAF. Intrathalamic gain at T1 explained a 587 significant amount of variance in T3 IAF $\left(\mathrm{R}^{2}=0.87, \mathrm{~F}(2,41)=138.65, \mathrm{p}<0.001\right)$. The 588 addition of $\mathrm{T} 3$ intrathalamic gain parameter did not significantly add to the explained 589 model variance $\left(\Delta \mathrm{R}^{2}=0.006, \Delta \mathrm{F}(1,40)=1.9, \mathrm{p}=0.175\right)$.

590 In a second multiple regression, we again included IAF at T3 as the dependent

591 variable and IAF and corticothalamic delay at $\mathrm{T} 1$ as predictors to account for baseline

592 levels before training. These predictors explained a significant amount of variance in T3

$593 \operatorname{IAF}\left(\mathrm{R}^{2}=0.87, \mathrm{~F}(2,41)=137.73, \mathrm{p}<0.001\right)$. Contrary to the prior analyses, the addition

594 of the T3 corticothalamic delay value significantly improved the explained model

595 variance $\left(\Delta \mathrm{R}^{2}=0.042, \Delta \mathrm{F}(1,40)=19.16, \mathrm{p}<0.001\right)$. Altogether, collapsed across the 
596 retreats, reductions in IAF were associated with increases in the corticothalamic delay

597 parameter $(\beta=-0.46)$.

598

\section{4. Discussion}

600 This paper presents the first mean-field computational model of scalp-measured

601 electrophysiology obtained during the practice of focused attention meditation. By

602 simulating and fitting EEG data recorded during meditation, this approach permits

603 mathematical analysis of longitudinal changes in oscillatory activity and makes testable

604 predictions to advance experimental research in this area. The mean-field modeling

605 approach of Robinson and colleagues (2001) was used to simulate scalp-recorded EEG

606 with the incorporation of corticocortical, corticothalamic, and intrathalamic loops. This

607 model was successfully extended to allow for longitudinal analysis of changes in model

608 parameters and intra-TRN connectivity due to training. We observed reliable training-

609 related changes in two model parameters, replicated for each of two separate training

610 groups. First, the intrathalamic gain parameter $\left(\mathrm{G}_{\mathrm{srs}}\right)$ decreased significantly with

611 training, suggesting reduced inhibition of modeled SRN cells by the TRN. Using stability

612 analysis, we found that the reduction in intrathalamic gain provided increased stability to

613 the modeled dynamical system. Second, the corticothalamic delay parameter $\left(\mathrm{t}_{0}\right)$

614 increased with training, indicating an increase in transmission delay between modeled

615 cortical and thalamic cells. The increase in modeled corticothalamic delay strongly

616 predicted individual reductions in experimentally observed individual alpha frequency

617 (Saggar et al., 2012). Lastly, intra-TRN connectivity analysis implicated a clear anterior-

618 posterior connectivity-based segregation in the modeled TRN layer. While this anterior- 
619 posterior connectivity remained stable over training assessments, an increase in between-

620 cluster anterior-posterior connectivity was observed in both retreat groups following

621 training. Taken together, these findings suggest that changes in attentional and other

622 cognitive processes through intensive meditation training may be supported by

623 longitudinal changes in corticothalamic dynamics.

624

625 4.1 Modeling Dense-array EEG with ER-MFM

626 To minimize the effect of local dependency at low frequencies, we restricted the analysis

627 to frequencies higher than $2 \mathrm{~Hz}$. Further, two specific methods were used to minimize the

628 local dependence effects at the alpha frequency. First, during preprocessing, EEG data

629 were transformed using scalp current density (SCD) estimation. The SCD transformation

630 creates reference-free EEG and reduces effects of volume conduction (Srinivasan et al.,

631 2007), thereby lowering the effect of distant activity on local sites. Second, we used a

632 nonparametric cluster-based permutation approach to identify longitudinal changes in

633 model parameters. This cluster-based approach is similar to cluster-corrected algorithms

634 used in fMRI data analysis, where information from neighboring voxels is considered

635 when estimating the activity at a local site (Smith \& Nichols, 2009). This approach,

636 although insensitive to extremely local changes (e.g., when studying effects at the single

637 scalp electrode level), works well for EEG data (Maris \& Oostenveld, 2007). Thus, by

638 including information about neighboring sites, this cluster-based extension helps to

639 minimize violation of local independence and likely provides a more plausible model

640 than the LEV approach alone. In the future, this approach should be tested against more 
641 sophisticated modeling approaches, where inter-connectivity between channels is also

642 modeled (O'Connor \& Robinson, 2004; Robinson et al., 2003).

643 After fitting the experimental EEG data, the R-MFM model was able to explain

644 more than $98 \%$ of the variance across channels, assessments, and groups (Table 2). Here,

645 SOBI and SMART were used to ensure that only high-quality EEG, relatively free from

646 EMG and EOG contamination, was used for model fitting (Saggar et al., 2012). This

647 likely contributed to the success of fitting the model to the recorded data. It is possible,

648 however, to further improve accuracy with more sophisticated fitting algorithms. For

649 example, Kerr and colleagues (2011) used Monte Carlo simulations to enable multiple

650 random initializations for data fitting, thereby reducing potential bias that can be

651 introduced when initial parameter values are held fixed.

652

\subsection{Longitudinal Changes in Intrathalamic Gain}

654 Following training, we observed a reduction in the amplitude of intrathalamic gain in the

655 modeled EEG sites overlying the right parietal region in both retreat groups. Compared to

656 pre-training values, intrathalamic gain was reduced by the mid-assessment point and

657 remained low at post-assessment. This parameter represents the strength of inhibitory

658 connection between the TRN and SRN cells. Because TRN cells exert a solely inhibitory

659 influence on the SRN in this model, the observed reduction in this parameter suggests

660 decreased inhibition of SRN cells.

661 The TRN has long been hypothesized to play important roles in both selective

662 attention (Crick, 1984) and general alertness (Steriade et al., 1993). Crick (1984) argued

663 that, during selective attention, TRN cell activity modulates the corticothalamic inputs 
664 that excite or inhibit specific SRN cells, thereby regulating the flow of sensory

665 information to the cortex. This hypothesized modulation of SRN cells is assumed to form

666 a reciprocal relation between the activity of TRN and SRN cells. In recent work,

667 McAlonan et al. (2008) provided direct evidence for this reciprocal relation by

668 demonstrating that TRN cells serve as an initial source of modulation in SRN cells

669 (McAlonan et al., 2008). In another study, Lam and Sherman (2011) reported that a

670 surprising percentage ( $25 \%)$ of the recorded neurons from somatosensory TRN received

671 non-topographic input from all three somatosensory relay nuclei of the thalamus (i.e.,

672 posterior medial, ventroposterior medial, and ventroposterior lateral relay nuclei),

673 suggesting that some subpopulations of TRN neurons may integrate somatosensory

674 inputs from different thalamic relays. The authors argued that, consistent with Crick's

675 hypothesis, such integration in TRN allows for competition between different inputs at

676 the thalamic level itself, allowing stronger and more salient inputs to suppress weaker and

677 less relevant inputs. Altogether, these and other related studies (Jones et al., 2010; Lam \&

678 Sherman, 2010), provide evidence for TRN's complex and versatile role in tuning

679 sensory inputs to enable salient information to reach the cortex.

680 Regarding the role of TRN in general alertness, Halassa et al. (2011) used

681 optogenetics and multi-electrode recording in awake behaving as well as sleeping mice to

682 selectively and causally test the role of the TRN in thalamic bursting and subsequent

683 generation of neocortical spindles. The authors reported that optogenetic activation of

684 TRN resulted in sleep-like spindles and that induction was state dependent and was

685 evident largely during non-rapid eye-movement (NREM) sleep and somewhat during 
686 wakefulness, but not at all during rapid eye-movement (REM) sleep (Halassa et al., 687 2011).

688 Taken together, this overview of TRN findings suggests that the reduction in 689 amplitude of the intrathalamic gain parameter (or inhibition by TRN cells) is consistent 690 with the notion that, following intensive training, practitioners may be more focused and 691 alert when engaged in meditation. This model-generated hypothesis provides some new 692 insight into the brain processes potentially associated with meditation training.

693 Specifically, modulation by the TRN cells could be responsible for enhanced cortical 694 activity for efficient processing of salient stimuli (i.e., object of focus) during meditation. 695 In support of this hypothesis, we have observed decreased bilateral frontocentral EEG 696 beta activity (1.2*IAF to $30 \mathrm{~Hz})$ in this same cohort of participants during practice of FA 697 meditation. Reduced beta activity suggests increased activation of fronto-parietal 698 attention networks with increased training in these techniques (Saggar et al 2012).

699 Behavioral evidence further indicated improved performance on tasks assessing visual 700 perception, vigilance, and response inhibition for this cohort following training (MacLean 701 et al., 2010; Sahdra et al., 2011). In order to directly examine the significance of the 702 intrathalamic gain parameter for models of enhanced attention and alertness, future work

703 should examine whether similar patterns of change in model parameters are observed 704 during performance of sustained attention and response inhibition tasks.

705 Interestingly, using a similar computational model based on only nine EEG

706 electrodes, Rowe et al. (2005) showed that the baseline intrathalamic gain parameter is

707 reduced in adolescents diagnosed with attention-deficit hyperactivity disorder (ADHD)

708 after stimulant medication. Hence, our modeling results can serve to motivate further 
709 study of shamatha and focused-attention meditation as part of a treatment approach for

710 patients with ADHD. Preliminary support for the efficacy of meditation training in

711 individuals with ADHD has already been shown in several recent studies incorporating

712 regular practice of FA meditation as a part of training (Mitchell et al., 2013; Zylowska et

713 al., 2008; 2009). Zylowska and colleagues (2008) conducted a study that included both

714 adolescents and adults with ADHD and reported post-training improvements in self-

715 reported scores on ADHD, depression, and anxiety symptoms, as well as behavioral

716 performance on attention regulation tasks. Further, these improvements were sustained at

717 a 3-month follow-up (Zylowska et al., 2009). In a recent pilot study, adults with ADHD

718 improved in self-reported and clinician ratings of ADHD, as well as task-based executive

719 functioning scores, following an 8-week group-based meditation training, as compared to

720 a randomized wait-list control group (Mitchell et al., 2013).

721

7224.3 Longitudinal Changes in Corticothalamic Delay

723 A corticothalamic delay parameter was incorporated in the model to represent the

724 conduction delay in signal transmission both from the cortex to thalamic nuclei and from

725 thalamic nuclei to the cortex. It is important to note that neither the R-MFM nor the

726 extended model presented in this paper presume existence of cortical or subcortical

727 pacemakers or "clocks" to generate periods of alpha and beta rhythms. Instead, the

728 corticothalamic delay itself facilitates emergence of alpha and beta rhythms on the

729 modeled scalp. Further, keeping all the other model parameters constant, the main effect

730 of increasing the corticothalamic delay results in reduced alpha and beta frequencies

731 (Robinson et al., 2001a; Saggar, 2011). Individual differences in this delay are also 
732 conjectured to contribute to the widely observed finding of individual differences in alpha

733 frequency (IAF) values (Robinson et al., 2001b). Other, more recent, computational

734 approaches have shown that the corticothalamic feed-forward vs. feedback delay (i.e.

735 asynchronicity) results in the emergence of surface-recorded alpha/beta rhythms without

736 using explicit pacemakers (Jones et al., 2009; Kerr et al., 2013). Thus, there appears to be

737 a strong basis for relating longitudinal changes in the modeled corticothalamic delay

738 parameter to previously reported findings of lowered IAF in these same participants

739 (Saggar et al 2012).

740 The model architecture used in this paper appears to replicate critical aspects

741 known from neurophysiological observation of the neuronal basis of alpha/beta rhythm

742 generation resulting from thalamocortical interactions. For instance, to understand the

743 neural basis of alpha-rhythm generation, Bollimunta and colleagues (2011) recently

744 concluded that the alpha generators in layer 6 of the macaque primary-visual cortex were

745 consistently the strongest contributors to alpha-rhythm generation across other

746 penetrations. This observation, along with previous results showing that feedback from

747 the striate cortex to thalamic nuclei originates from layer 6 of the same cortical column

748 that receives afferents from the thalamic nuclei (Callaway, 1998; Sherman \& Guillery,

749 2006), supports the idea that the alpha rhythm recorded over primary visual cortex of

750 macaques could be generated primarily by thalamocortical interactions (Bollimunta et al., $7512011)$

752 Here, we observed an increase in the model parameter representing

753 corticothalamic delay in modeled electrodes overlaying parietal-occipital regions. The

754 increase in corticothalamic delay was observed across both training groups, and was 
755 evident at both the mid- and post-assessment points. Importantly, the increase in modeled

756 delay values predicted a drop in observed alpha frequency (IAF values) after training.

757 Studies of meditation, including our previous work (Saggar et al. 2012), have repeatedly

758 shown that long-term meditators have reduced alpha frequency during meditation

759 (Banquet, 1973; Kasamatsu \& Hirai, 1966; Wallace, 1970; Zhang, 1988) as well as

760 during baseline rest (Aftanas \& Golocheikine, 2002; 2001; Aftanas \& Golocheikine,

761 2003). However, the biological basis for this finding is still unknown.

762 The present modeling results suggest that the increased corticothalamic delay

763 involving parietal-occipital regions might account for the reduced alpha frequency

764 observed in meditators. However, the construct of "corticothalamic delay" itself is

765 complex. Multiple potential processes can influence corticothalamic effective delay.

766 These include variable synaptic delays, activation of different subpopulations of

767 pyramidal cells, interareal activation sequences, and changes in axonal and dendritic

768 cable properties due to myelination variations (Meeren et al., 2002). Further, in addition

769 to corticothalamic delay, changes in axonal conductance and decay time (anesthesia

770 induced) have also been reported as a putative cause for reduced alpha (or dominant)

771 frequency (Ching et al., 2010; Englehardt et al., 1991; Nunez, 1995). Additionally,

772 changes in the dendritic decay rate have also been shown to affect alpha frequency

773 (Robinson et al., 2001). As no longitudinal changes were observed in the dendritic decay

774 rate parameter, changes in the corticothalamic delay parameter seem the most viable

775 candidate for reduction in alpha frequency following meditation training. However, it is

776 unclear as to why an increase in posterior corticothalamic delay might occur with

777 intensive meditation training. 
779 corticothalamic delay is to facilitate sustained attention on incoming sensory or tactile

780 stimuli with increased meditative expertise. This idea is based upon the argument that an

781 increased delay will facilitate a subsequent increased asynchrony between the firing rates

782 of the cortical and thalamic cells, potentially disrupting ongoing corticothalamic

783 synchronization. In line with this argument, similar asynchronous inputs have been

784 shown to be responsible for weakening previously strengthened inputs in the

785 hippocampal cells in rats (Csicsvari et al., 2003; Huerta \& Lisman, 1995). Given the

786 regulatory role of TRN, the transient disruption of corticothalamic synchronization may

787 enable increased effective SRN-based activation of cortex and thus modulate the initial

788 processing of sensory inputs.

789

\section{$790 \quad 4.4$ Stability Analysis}

791 Nonlinear instabilities or bifurcations in large-scale dynamical systems have been of wide

792 interest to computational scientists. A sudden change in large-scale dynamical systems

793 activity is considered to be a bifurcation (e.g., a transition from laminar to turbulent fluid

794 flow is a common example of such change; Breakspear et al., 2006)). Due to their

795 disruptive nature for brain function, it is especially important to study such instabilities in 796 neural dynamics (e.g., onset of seizure; Le Van Quyen et al., 2003; Mirowski et al., 797 2009)). Robinson et al. (2002) extensively explored the dynamics of their R-MFM 798 approach and observed that the model had only a few key instabilities that result in 799 nonlinear behaviors. Of these instabilities, the first two (known as slow-wave and theta) 800 lead to spike-wave nonlinear limit cycles in the slow-delta and fast-theta ranges, 
801 respectively. The other two instabilities (spindle and alpha peak) lead to a limit cycle

802 with frequencies in alpha band range $(\sim 10 \mathrm{~Hz})$. The spindle instability is of particular

803 interest to our work as it originates in the intrathalamic loop (where we observed effects

804 of meditation training) and spreads to the cortex due to thalamocortical projections

805 (Breakspear et al., 2006; Robinson et al., 2002).

806 Using the reduced 3-d stability space, we observed that participants in both retreat

807 groups had a significantly lower z-coordinate (representing intrathalamic stability)

808 following training. This suggests that retreat participants' modeled brain state was further

809 below the intrathalamic instability boundary (or tent's surface in $\mathrm{z}$ direction) after

810 training. This decrease in the z-coordinate value is likely due to the observed decrease in

811 the model's intrathalamic gain after training (see section 2.10).

812 Although the participants in this study were neurologically healthy individuals,

813 increased distance from the modeled intrathalamic instability boundary due to meditation

814 training provides a fascinating motivation to further study shamatha and related focused-

815 attention meditation as part of potential treatment strategies for epileptic patients. Recent

816 reviews of psychobehavioral interventions for persons with epilepsy conceptualize

817 interictal periods as a time-varying pattern of changing proximity to seizure boundary

818 conditions (Tang et al., 2014). Thus, even modest improvements in stability, such as

819 those reported here, may become critical in delaying or preventing seizure onset when an

820 individual approaches the seizure boundary.

821

8224.5 Connectivity in TRN layer 
823 To model intra-TRN connectivity, a novel computational procedure allowed us to explore

824 the connectivity structure without fitting a myriad of additional model parameters. Using

825 this method, a clear frontoparietal functional division was observed in the modeled TRN

826 layer. Interestingly, the discovery of a functional division in the modeled TRN layer is in

827 line with animal studies where the afferent cortical connections to TRN layer are shown

828 to be topographically structured (Zikopoulos \& Barbas, 2007). Although this functional

829 division was stable across time points, an increase in anterior-posterior between-cluster

830 connectivity associated with meditation training was observed in both retreat groups. This

831 effect was only evident for the post time point in RG1 and for the middle time point in

832 RG2. One plausible reason for lack of consistency across retreats could be the small

833 sample size of our study.

$834 \quad$ Using animal models, it has been well established that the cortical and thalamic

835 afferents to the TRN are topographically organized (Crabtree \& Killackey, 1989). Along

836 the rostrocaudal axis, centroposterior loci in TRN receive afferents from the

837 somatosensory, visual, and auditory cortices and their associated thalamic relay nuclei.

838 Recent work by Zikopoulos and Barbas (2006), however, has provided novel evidence of

839 strong prefrontal projections in the anterior sector of TRN. These authors have claimed

840 that these prefrontal projections, although concentrated in the anterior TRN sector, are

841 widespread and overlap extensively with the afferent projections from other cortical and

842 thalamic pathways in the TRN (Zikopoulos \& Barbas, 2006). Additionally, the overlap

843 between prefrontal and other cortical/thalamic projections in the TRN is thought to

844 contribute to attentional selection of salient stimuli and attentional regulation (Zikopoulos

845 \& Barbas, 2007). 
846 In light of this research, it could be speculated that the increase in connectivity

847 between the anterior and posterior clusters in the modeled TRN layer following

848 meditation training could be due to increased prefrontal projection strength on the TRN

849 layer. This may reflect a putative mechanism for facilitating sustained attention on the

850 incoming sensory/tactile breath stimulation. In this same cohort of participants, enhanced

851 attention regulation as evidenced by improved response inhibition (presumed to be a

852 frontally-mediated component of executive control) was observed following meditation

853 training (Sahdra et al., 2011). Utilizing the ER-MFM model extension, future research

854 can investigate this hypothesis by modeling EEG data collected while participants are

855 engaged in an attention regulation task.

856

857 4.6 Limitations

858 Several limitations should be discussed. Our modeling approach was restricted to cortico-

859 cortical, cortico-thalamo-cortical, and intrathalamic interactions. While these interactions

860 are known to efficiently capture the general context of arousal and attention regulation,

861 activity in other subcortical areas (e.g., the hippocampus and amygdala) are also known

862 to be modulated during meditation practice (for a recent review, see Fox et al., (2014)).

863 Future studies could examine whether the approach taken here can be adapted to include

864 additional subcortical areas and associated interactions. This model may therefore be

865 further extended to better understand the hypothesized mechanisms underlying shamatha

866 and FA meditation practice and training.

867 Several factors unrelated to meditation training could have also contributed to the

868 present findings. First, both retreats took place in a remote wilderness setting, where 
869 participants spent a majority of their time in solitary meditation. The environmental and

870 acute behavioral changes associated with substantial time in solitary meditation likely

871 exerted a number of non-specific effects. These could have manifested as observed

872 physiological changes that were reflected in the model parameters. Second, the amount of

873 motivation may not have been matched between the initial retreat and wait-list control

874 groups, as the participants were aware of their group assignment prior to receiving

875 meditation training. Although our wait-list design likely addressed several important

876 design limitations of prior research on intensive meditation, future investigations should

877 attempt to better account for various social, motivational, and environmental factors by

878 using active control conditions or by comparing training periods of differing lengths.

879 Other limitations of this work come from the sparseness of cortical sampling (i.e.,

880 number of EEG sensors used and modeled). One future avenue of research could be to

881 concurrently use high spatial resolution neuroimaging modalities (e.g., fMRI) for better

882 modeling and understanding of neural data collected during meditation practice.

883 Another potential limitation concerns the quantification of IAF values. To

884 calculate IAF values for each participant, we employed the widely used power-weighted

885 (or center of gravity) method to determine mean alpha frequency across all channels

886 (Klimesch, 1999). This power-weighted method provides an advantage over visual

887 inspection based methods to find the peak alpha frequency: in cases where the EEG

888 spectra has multiple peaks or is flat in the alpha range, power-weighted methods better

889 reflect the central tendency of alpha power and are more representative of the underlying

890 activity (Goljahani et al., 2012). However, to calculate power-weighted IAF, a

891 predetermined interval of alpha frequencies is required. Defining such a priori intervals 
892 can lead to biased estimates, particularly when participants have a very high or low alpha

893 peak. Thus, new automated methods, based on regression (Chiang et al., 2008) and

894 channel selection (Goljahani et al., 2012) have been recently proposed. Future work is

895 thus required to carefully examine how the calculation of IAF using novel methods may

896 influence the assessment of IAF values in long-term meditators.

897 Lastly, it is important to note that the modeling approach presented here, although

898 widely accepted in the extant literature related to EEG-based computational models, is

899 still very approximate in nature and is based on a number of assumptions. These

900 assumptions are required to generate models that are computationally practical and

901 tractable. We argue that such modeling approaches, while not mechanistically accurate,

902 may still enable progress in understanding complex brain phenomenon. By simulating a

903 complex phenomenon (or associated neurophysiological correlates) based on an initial

904 formulation, the goal of such approaches is to generate novel hypotheses that may

905 motivate future research studies and data collection, and in turn refine the model and its

906 underlying assumptions. Such a process implements the classic cycle of theory

907 development, testing, and revision to advance our understanding.

908

9094.7 Conclusions and Future work

910 We present the first computational model of neurophysiological changes during shamatha

911 and related FA meditation, which advances our previous work investigating scalp-

912 recorded EEG during meditation by examining the putative role of corticothalamic and

913 intrathalamic interactions in observed longitudinal changes in cortical activity associated

914 with intensive meditation training. Our computational approach offers several testable 
915 hypotheses that could motivate the incorporation of shamatha and FA meditation in novel

916 treatment approaches. Specifically, the observed decreased in intrathalamic gain

917 parameter following training serves to motivate further study of shamatha and FA

918 meditation as a potential treatment approach for individuals with ADHD, whereas

919 increased model stability after training motivates further analysis of the potential efficacy

920 of shamatha and FA meditation practice as part of treatment strategies for neurological

921 disorders (e.g., epilepsy). Finally, a similar modeling approach can be applied to EEG

922 data obtained during performance of cognitive tasks so that changes in model parameters

923 can be directly related to training-related changes in measureable task performance.

924

925 
927 This work was supported by Fetzer Institute Grant \#2191, John Templeton Foundation

928 Grant 39970 to Clifford D. Saron, and by gifts from the Hershey Family, Chade-Meng Institute for Consciousness Studies, the Baumann Foundation, Grant Couch and Louise Pearson, Caroline Zecca-Ferris and anonymous, and other individual donors, all to Clifford D. Saron. The work was additionally supported by a F. J. Varela research award from the Mind and Life Institute to Manish Saggar and a National Science Foundation predoctoral fellowship to Katherine A. MacLean. Sponsorship in the form of publicity for participant recruitment and discount services were provided by the Shambhala Mountain Center and in the form of an equipment loan by the Mind and Life Institute.

\section{References:}

Aftanas, L.I., Golosheikin, S.A., 2001. Human anterior and frontal midline theta and lower alpha reflect emotionally positive state and internalized attention: highresolution EEG investigation of meditation. Neurosci Lett 310, 57-60.

Aftanas, L.I., Golosheikin, S.A., 2002. Non-linear dynamic complexity of the human EEG during meditation. Neurosci Lett 330, 143-146.

Aftanas, L.I., Golosheikin, S.A., 2003. Changes in cortical activity in altered states of consciousness: The study of meditation by high-resolution EEG. Human Physiology $29,143-151$.

Austin, J.H., 2013. Zen and the brain: mutually illuminating topics. Front Psychol 4, 784. doi:10.3389/fpsyg.2013.00784

Banquet, J.P., 1973. Spectral analysis of the EEG in meditation. Electroencephalography and clinical Neurophysiology 35, 143-151.

Belouchrani, A., Abed-Meraim, K., Cardoso, J.F., Moulines, E., 1993. Second-order blind separation of temporally correlated sources, in:. Presented at the Proc. Int. Conf. Digital Signal Processing, pp. 346-351.

Bollimunta, A., Mo, J., Schroeder, C.E., Ding, M., 2011. Neuronal mechanisms and attentional modulation of corticothalamic $\alpha$ oscillations. Journal of Neuroscience 31, 4935-4943. doi:10.1523/JNEUROSCI.5580-10.2011

Breakspear, M., Roberts, J.A., Terry, J.R., Rodrigues, S., Mahant, N., Robinson, P.A., 2006. A unifying explanation of primary generalized seizures through nonlinear brain modeling and bifurcation analysis. Cerebral Cortex 16, 1296.

Brefczynski-Lewis, J.A., Lutz, A., Schaefer, H.S., Levinson, D.B., Davidson, R.J., 2007. Neural correlates of attentional expertise in long-term meditation practitioners. Proceedings of the National Academy of Sciences 104, 11483.

Bressler, S.L., Menon, V., 2010. Large-scale brain networks in cognition: emerging methods and principles. Trends in Cognitive Sciences 14, 277-290. doi:10.1016/j.tics.2010.04.004

Cahn, B.R., Polich, J., 2006. Meditation states and traits: EEG, ERP, and neuroimaging studies. Psychol Bull 132, 32.

Callaway, E.M., 1998. Local circuits in primary visual cortex of the macaque monkey. Annu Rev Neurosci 21, 47-74. doi:10.1146/annurev.neuro.21.1.47 
971 Chiang, A.K.I., Rennie, C.J., Robinson, P.A., Roberts, J.A., Rigozzi, M.K., Whitehouse,

972 R.W., Hamilton, R.J., Gordon, E., 2008. Automated characterization of multiple

973 alpha peaks in multi-site electroencephalograms. J. Neurosci. Methods 168, 396-411.

974

975

976

977

978

979

980

981

982

983

984

985

986

987

988

989

990

991

992

993

994

995

996

997

998

999

1000

1001

1002

1003

1004

1005

1006

1007

1008

1009

1010

1011

1012

1013

1014

1015 doi:10.1016/j.jneumeth.2007.11.001

Ching, S., Cimenser, A., Purdon, P.L., Brown, E.N., Kopell, N.J., 2010. Thalamocortical model for a propofol-induced alpha-rhythm associated with loss of consciousness. Proceedings of the National Academy of Sciences 107, 22665-22670. doi:10.1073/pnas.1017069108

Coleman, T.F., Li, Y., 1993. An interior trust region approach for nonlinear minimization subject to bounds. SIAM Journal on Optimization.

Corbetta, M., Shulman, G.L., 2002. Control of goal-directed and stimulus-driven attention in the brain. Nat. Rev. Neurosci. 3, 201-215. doi:10.1038/nrn755

Crabtree, J.W., Killackey, H.P., 1989. The topographic organization and axis of projection within the visual sector of the rabbit's thalamic reticular nucleus. European Journal of Neuroscience 1, 94-109.

Crick, F., 1984. Function of the thalamic reticular complex: the searchlight hypothesis. Proc. Natl. Acad. Sci. U.S.A. 81, 4586.

Csicsvari, J., Jamieson, B., Wise, K.D., Buzsaki, G., 2003. Mechanisms of gamma oscillations in the hippocampus of the behaving rat. Neuron 37, 311-322.

Dosenbach, N.U.F., Fair, D.A., Cohen, A.L., Schlaggar, B.L., Petersen, S.E., 2008. A dual-networks architecture of top-down control. Trends in Cognitive Sciences 12, 99-105. doi:10.1016/j.tics.2008.01.001

Englehardt, W., Carl, G., Dierks, T., Maurer, K., 1991. Electroencephalographic mapping during isoflurane anesthesia for treatment of mental depression. J Clin Monit 7, 2329.

Fan, J., McCandliss, B.D., Fossella, J., Flombaum, J.I., Posner, M.I., 2005. The activation of attentional networks. NeuroImage 26, 471-479. doi:10.1016/j.neuroimage.2005.02.004

Fox, K.C.R., Nijeboer, S., Dixon, M.L., Floman, J.L., Ellamil, M., Rumak, S.P., Sedlmeier, P., Christoff, K., 2014. Is meditation associated with altered brain structure? A systematic review and meta-analysis of morphometric neuroimaging in meditation practitioners. Neurosci Biobehav Rev. doi:10.1016/j.neubiorev.2014.03.016

Freeman, W.J., 1972. Linear analysis of the dynamics of neural masses. Annual Review of Biophysics and Bioengineering 1, 225-256.

Freeman, W.J., 1975. Mass action in the nervous system. Academic Press New York.

Freeman, W.J., 1987. Simulation of chaotic EEG patterns with a dynamic model of the olfactory system. Biol Cybern 56, 139-150.

Friedman, M., 1937. The use of ranks to avoid the assumption of normality implicit in the analysis of variance. Journal of the American Statistical Association 32, 675-701.

Goldman, R.I., Stern, J.M., Engel, J., Jr, Cohen, M.S., 2002. Simultaneous EEG and fMRI of the alpha rhythm. NeuroReport 13, 2487.

Goljahani, A., D'Avanzo, C., Schiff, S., Amodio, P., Bisiacchi, P., Sparacino, G., 2012. A novel method for the determination of the EEG individual alpha frequency. NeuroImage 60, 774-786. doi:10.1016/j.neuroimage.2011.12.001 
1017

1018

1019

1020

1021

1022

1023

1024

1025

1026

1027

1028

1029

1030

1031

1032

1033

1034

1035

1036

1037

1038

1039

1040

1041

1042

1043

1044

1045

1046

1047

1048

1049

1050

1051

1052

1053

1054

1055

1056

1057

1058

1059

1060

1061

1062

Guglietti, C.L., Daskalakis, Z.J., Radhu, N., Fitzgerald, P.B., Ritvo, P., 2012. Meditationrelated increases in GABAB modulated cortical inhibition. Brain Stimulation 1-6. doi:10.1016/j.brs.2012.08.005

Halassa, M.M., Siegle, J.H., Ritt, J.T., Ting, J.T., Feng, G., Moore, C.I., 2011. Selective optical drive of thalamic reticular nucleus generates thalamic bursts and cortical spindles. Nat. Neurosci. 14, 1118-1120. doi:10.1038/nn.2880

Hasenkamp, W., Barsalou, L.W., 2012. Effects of meditation experience on functional connectivity of distributed brain networks. Front Hum Neurosci 6, 38 . doi:10.3389/fnhum.2012.00038

Hasenkamp, W., Wilson-Mendenhall, C.D., Duncan, E., Barsalou, L.W., 2012. Mind wandering and attention during focused meditation: a fine-grained temporal analysis of fluctuating cognitive states. NeuroImage 59, 750-760. doi:10.1016/j.neuroimage.2011.07.008

Hölzel, B.K., Carmody, J., Vangel, M., Congleton, C., Yerramsetti, S.M., Gard, T., Lazar, S.W., 2011. Mindfulness practice leads to increases in regional brain gray matter density. Psychiatry Res 191, 36-43. doi:10.1016/j.pscychresns.2010.08.006

Huerta, P.T., Lisman, J.E., 1995. Bidirectional synaptic plasticity induced by a single burst during cholinergic theta oscillation in CA1 in vitro. Neuron 15, 1053-1063.

Isaksson, A., Wennberg, A., Zetterberg, L.H., 1981. Computer analysis of EEG signals with parametric models. Proceedings of the IEEE 69, 451-461.

Jha, A.P., Krompinger, J., Baime, M.J., 2007. Mindfulness training modifies subsystems of attention. Cogn Affect Behav Neurosci 7, 109-119.

Jirsa, V.K., Sporns, O., Breakspear, M., Deco, G., McIntosh, A.R., 2010. Towards the virtual brain: network modeling of the intact and the damaged brain. Arch Ital Biol 148, 189-205.

Jones, E.G., 2009. Synchrony in the interconnected circuitry of the thalamus and cerebral cortex. Ann. N. Y. Acad. Sci. 1157, 10-23. doi:10.1111/j.1749-6632.2009.04534.x

Jones, S.R., Kerr, C.E., Wan, Q., Pritchett, D.L., Hämäläinen, M., Moore, C.I., 2010. Cued spatial attention drives functionally relevant modulation of the mu rhythm in primary somatosensory cortex. Journal of Neuroscience 30, 13760-13765. doi:10.1523/JNEUROSCI.2969-10.2010

Jones, S.R., Pritchett, D.L., Sikora, M.A., Stufflebeam, S.M., Hämäläinen, M., Moore, C.I., 2009. Quantitative Analysis and Biophysically Realistic Neural Modeling of the MEG Mu Rhythm: Rhythmogenesis and Modulation of Sensory-Evoked Responses. J Neurophysiol 102, 3554-3572. doi:10.1152/jn.00535.2009

Jones, S.R., Pritchett, D.L., Stufflebeam, S.M., Hämäläinen, M., Moore, C.I., 2007. Neural correlates of tactile detection: a combined magnetoencephalography and biophysically based computational modeling study. 27, 10751-10764. doi:10.1523/JNEUROSCI.0482-07.2007

Kabat-Zinn, J., 1994. Wherever you go, there you are: Mindfulness meditation in everyday life. Hyperion Books.

Kasamatsu, A., Hirai, T., 1966. An electroencephalographic study on the Zen meditation (Zazen). Psychiatry Clin Neurosci 20, 315-336.

Kastner, S., Saalmann, Y.B., Schneider, K.A., 2012. Thalamic Control of Visual Attention. Oxford University Press. doi:10.1093/acprof:oso/9780195334364.003.0003 
1063

1064

1065

1066

1067

1068

1069

1070

1071

1072

1073

1074

1075

1076

1077

1078

1079

1080

1081

1082

1083

1084

1085

1086

1087

1088

1089

1090

1091

1092

1093

1094

1095

1096

1097

1098

1099

1100

1101

1102

1103

1104

1105

1106

1107

1108

Kayser, J., Tenke, C.E., 2006. Principal components analysis of Laplacian waveforms as a generic method for identifying ERP generator patterns: I. Evaluation with auditory oddball tasks. Clinical neurophysiology 117, 348-368.

Kerr, C.C., Rennie, C.J., Robinson, P.A., 2008. Physiology-based modeling of cortical auditory evoked potentials. Biol Cybern 98, 171-184.

Kerr, C.C., Rennie, C.J., Robinson, P.A., 2011. Model-based analysis and quantification of age trends in auditory evoked potentials. Clinical neurophysiology 122, 134-147.

Kerr, C.C., van Albada, S.J., Rennie, C.J., Robinson, P.A., 2010. Age trends in auditory oddball evoked potentials via component scoring and deconvolution. Clinical neurophysiology 121, 962-976.

Kerr, C.E., Sacchet, M.D., Lazar, S.W., Moore, C.I., Jones, S.R., 2013. Mindfulness starts with the body: somatosensory attention and top-down modulation of cortical alpha rhythms in mindfulness meditation. Front Hum Neurosci 7, 12. doi:10.3389/fnhum.2013.00012

Klimesch, W., 1999. EEG alpha and theta oscillations reflect cognitive and memory performance: a review and analysis. Brain Research Reviews 29, 169-195.

Lagerlund, T.D., Sharbrough, F.W., 1989. Computer simulation of the generation of the electroencephalogram. Electroencephalography and clinical Neurophysiology 72, 31.

Langri, T. J., 2009. Is Meditation a Means of Knowing our Mental World? [WWW Document]. URL andrewmacnairarchitect.com/FRANK\%20BOOK/1.doc (accessed 7.15.14).

Lam, Y.-W., Sherman, S.M., 2010. Functional organization of the somatosensory cortical layer 6 feedback to the thalamus. Cerebral Cortex 20, 13-24. doi:10.1093/cercor/bhp077

Lam, Y.-W., \& Sherman, S. M. (2011). Functional organization of the thalamic input to the thalamic reticular nucleus. Journal of Neuroscience, 31(18), 6791-6799. doi:10.1523/JNEUROSCI.3073-10.2011

Larson, C.L., Davidson, R.J., Abercrombie, H.C., Ward, R.T., Schaefer, S.M., Jackson, D.C., Holden, J.E., Perlman, S.B., 1998. Relations between PET-derived measures of thalamic glucose metabolism and EEG alpha power. Psychophysiology 35, 162-169.

Le Van Quyen, M., Navarro, V., Martinerie, J., Baulac, M., Varela, F.J., 2003. Toward a neurodynamical understanding of ictogenesis. Epilepsia 44 Suppl 12, 30-43.

Liley, D., Wright, J.J., 1994. Intracortical connectivity of pyramidal and stellate cells: estimates of synaptic densities and coupling symmetry. Network: Computation in Neural Systems 5, 175-189.

Lopes da Silva, F.H., Hoeks, A., Smits, H., Zetterberg, L.H., 1974. Model of brain rhythmic activity. Biol Cybern 15, 27-37.

Lumer, E.D., Edelman, G.M., Tononi, G., 1997. Neural dynamics in a model of the thalamocortical system. I. Layers, loops and the emergence of fast synchronous rhythms. Cerebral Cortex 7, 207.

Lutz, A., Slagter, H.A., Dunne, J.D., Davidson, R.J., 2008. Attention regulation and monitoring in meditation. Trends in Cognitive Sciences 12, 163-169.

Lutz, A., Slagter, H.A., Rawlings, N.B., Francis, A.D., Greischar, L.L., Davidson, R.J., 2009. Mental Training Enhances Attentional Stability: Neural and Behavioral Evidence. Journal of Neuroscience 29, 13418.

MacLean, K.A., Ferrer, E., Aichele, S.R., Bridwell, D.A., Zanesco, A.P., Jacobs, T.L., 

G.R., Saron, C.D., 2010. Intensive meditation training improves perceptual discrimination and sustained attention. Psychol Sci 21, 829-839. doi:10.1177/0956797610371339

Maris, E., Oostenveld, R., 2007. Nonparametric statistical testing of EEG-and MEG-data. J. Neurosci. Methods 164, 177-190.

Maris, E., Schoffelen, J.M., Fries, P., 2007. Nonparametric statistical testing of coherence differences. J. Neurosci. Methods 163, 161-175.

MATLAB, 2010. The MathWorks Inc. Natick, MA.

McAlonan, K., Cavanaugh, J., Wurtz, R.H., 2008. Guarding the gateway to cortex with attention in visual thalamus. Nature 456, 391-394. doi:10.1038/nature07382

Meeren, H.K.M., Pijn, J.P.M., Van Luijtelaar, E.L.J.M., Coenen, A.M.L., Lopes da Silva, F.H., 2002. Cortical focus drives widespread corticothalamic networks during spontaneous absence seizures in rats. Journal of Neuroscience 22, 1480-1495.

Menon, V., 2011. Large-scale brain networks and psychopathology: a unifying triple network model. Trends in Cognitive Sciences 15, 483-506. doi:10.1016/j.tics.2011.08.003

Mirowski, P., Madhavan, D., Lecun, Y., Kuzniecky, R., 2009. Classification of patterns of EEG synchronization for seizure prediction. Clin Neurophysiol 120, 1927-1940. doi:10.1016/j.clinph.2009.09.002

Mitchell, J.T., McIntyre, E.M., English, J.S., Dennis, M.F., Beckham, J.C., Kollins, S.H., 2013. A Pilot Trial of Mindfulness Meditation Training for ADHD in Adulthood: Impact on Core Symptoms, Executive Functioning, and Emotion Dysregulation. J Atten Disord. doi: 10.1177/1087054713513328

Mitra, P.P., Pesaran, B., 1999. Analysis of dynamic brain imaging data. Biophysical journal 76, 691-708.

Moore, A., Gruber, T., Derose, J., Malinowski, P., 2012. Regular, brief mindfulness meditation practice improves electrophysiological markers of attentional control. Front Hum Neurosci 6, 18. doi:10.3389/fnhum.2012.00018

Newberg, AB, Iversen, J., 2003. The neural basis of the complex mental task of meditation: neurotransmitter and neurochemical considerations. Medical hypotheses 61, 282-291.

Nunez, P.L., 1974a. The brain wave equation: A model for the EEG. Mathematical Biosciences 21, 279-297.

Nunez, P.L., 1974b. Wavelike properties of the alpha rhythm. IEEE Transactions on Biomedical Engineering 473-482.

Nunez, P.L., 1995. Neocortical dynamics and human EEG rhythms. Oxford University Press, USA.

O'Connor, S.C., Robinson, P.A., 2004. Spatially uniform and nonuniform analyses of electroencephalographic dynamics, with application to the topography of the alpha rhythm. Phys. Rev. E 70, 11911.

Oostenveld, R., Fries, P., Maris, E., Schoffelen, J.M., 2011. FieldTrip: open source software for advanced analysis of MEG, EEG, and invasive electrophysiological data. Computational Intelligence and Neuroscience 2011, 1.

Perrin, F., Pernier, J., Bertrand, O., Echallier, J.F., 1989. Spherical splines for scalp potential and current density mapping. Electroencephalography and clinical 
1155

1156

1157

1158

1159

1160

1161

1162

1163

1164

1165

1166

1167

1168

1169

1170

1171

1172

1173

1174

1175

1176

1177

1178

1179

1180

1181

1182

1183

1184

1185

1186

1187

1188

1189

1190

1191

1192

1193

1194

1195

1196

1197

1198

1199

1200
Neurophysiology 72, 184-187.

Pizzagalli, D.A., 2007. Electroencephalography and high-density electrophysiological source localization. Handbook of psychophysiology 3, 56-84.

Reimann, M.W., Anastassiou, C.A., Perin, R., Hill, S.L., Markram, H., Koch, C., 2013. A biophysically detailed model of neocortical local field potentials predicts the critical role of active membrane currents. Neuron 79, 375-390. doi:10.1016/j.neuron.2013.05.023

Rennie, C.J., Robinson, P.A., Wright, J.J., 1999. Effects of local feedback on dispersion of electrical waves in the cerebral cortex. Phys. Rev. E 59, 3320-3329.

Rennie, C.J., Robinson, P.A., Wright, J.J., 2002. Unified neurophysical model of EEG spectra and evoked potentials. Biol Cybern 86, 457-471.

Robinson, P.A., Loxley, P.N., O'Connor, S.C., Rennie, C.J., 2001a. Modal analysis of corticothalamic dynamics, electroencephalographic spectra, and evoked potentials. Phys Rev E Stat Nonlin Soft Matter Phys 63, 041909.

Robinson, P.A., Rennie, C.J., Rowe, D.L., 2002. Dynamics of large-scale brain activity in normal arousal states and epileptic seizures. Phys. Rev. E 65, 41924.

Robinson, P.A., Rennie, C.J., Rowe, D.L., O'Connor, S.C., Wright, J.J., Gordon, E., Whitehouse, R.W., 2003. Neurophysical modeling of brain dynamics. Neuropsychopharmacology 28, 74.

Robinson, P.A., Whitehouse, R.W., Rennie, C.J., 2003. Nonuniform corticothalamic continuum model of electroencephalographic spectra with application to split-alpha peaks. Phys Rev E Stat Nonlin Soft Matter Phys 68, 021922.

Robinson, P.A., Rennie, C.J., Wright, J.J., 1997. Propagation and stability of waves of electrical activity in the cerebral cortex. Phys. Rev. E 56, 826-840.

Robinson, P.A., Rennie, C.J., Wright, J.J., Bahramali, H., Gordon, E., Rowe, D.L., 2001b. Prediction of electroencephalographic spectra from neurophysiology. Phys. Rev. E 63, 21903.

Robinson, P.A., Rennie, C.J., Wright, J.J., Bourke, P.D., 1998. Steady states and global dynamics of electrical activity in the cerebral cortex. Phys. Rev. E 58, 3557-3571.

Rowe, D.L., Robinson, P.A., Gordon, E., 2005. Stimulant drug action in attention deficit hyperactivity disorder (ADHD): inference of neurophysiological mechanisms via quantitative modelling. Clinical neurophysiology 116, 324-335.

Rowe, D.L., Robinson, P.A., Rennie, C.J., 2004a. Estimation of neurophysiological parameters from the waking EEG using a biophysical model of brain dynamics. Journal of theoretical biology 231, 413-433.

Rowe, D.L., Robinson, P.A., Rennie, C.J., Harris, A.W., Felmingham, K.L., Lazzaro, I.L., Gordon, E., 2004b. Neurophysiologically-based mean-field modelling of tonic cortical activity in post-traumatic stress disorder (PTSD), schizophrenia, first episode schizophrenia and attention deficit hyperactivity disorder (ADHD). Journal of integrative neuroscience $3,453$.

Saggar, M., 2011. Computational Analysis of Meditation. Retrieved from University of Texas Digital Repository: Electronic Theses and Dissertations, (Austin, TX).

Saggar, M., King, B.G., Zanesco, A.P., MacLean, K.A., Aichele, S.R., Jacobs, T.L., Bridwell, D.A., Shaver, P.R., Rosenberg, E.L., Sahdra, B.K., Ferrer, E., Tang, A.C., Mangun, G.R., Wallace, B.A., Miikkulainen, R., Saron, C.D., 2012. Intensive training induces longitudinal changes in meditation state-related EEG oscillatory 
1201

1202

1203

1204

1205

1206

1207

1208

1209

1210

1211

1212

1213

1214

1215

1216

1217

1218

1219

1220

1221

1222

1223

1224

1225

1226

1227

1228

1229

1230

1231

1232

1233

1234

1235

1236

1237

1238

1239

1240

1241

1242

1243

1244

1245

1246 activity. Front Hum Neurosci 6, 256. doi:10.3389/fnhum.2012.00256

Sahdra, B.K., MacLean, K.A., Ferrer, E., Shaver, P.R., Rosenberg, E.L., Jacobs, T.L., Zanesco, A.P., King, B.G., Aichele, S.R., Bridwell, D.A., Mangun, G.R., Lavy, S., Wallace, B.A., Saron, C.D., 2011. Enhanced response inhibition during intensive meditation training predicts improvements in self-reported adaptive socioemotional functioning. Emotion 11, 299-312. doi:10.1037/a0022764

Schreckenberger, M., Lange-Asschenfeldt, C., Lange-Asschenfeld, C., Lochmann, M., Mann, K., Siessmeier, T., Buchholz, H.-G., Bartenstein, P., Gründer, G., 2004. The thalamus as the generator and modulator of EEG alpha rhythm: a combined PET/EEG study with lorazepam challenge in humans. NeuroImage 22, 637-644. doi:10.1016/j.neuroimage.2004.01.047

Sherman, S.M., Guillery, R.W., 2006. Exploring the thalamus and its role in cortical function. MIT press Cambridge, MA.

Shwedyk, E., Balasubramanian, R., Scott, R.N., 1977. A nonstationary model for the electromyogram. IEEE Transactions on Biomedical Engineering 417-424.

Slagter, H.A., Lutz, A., Greischar, L.L., Francis, A.D., Nieuwenhuis, S., Davis, J.M., Davidson, R.J., 2007. Mental training affects distribution of limited brain resources. PLoS Biol. 5, e138.

Slagter, H. A., Davidson, R. J., \& Lutz, A. (2011). Mental Training as a Tool in the Neuroscientific Study of Brain and Cognitive Plasticity. Frontiers in Human Neuroscience, 5.

Smith, S.M., Nichols, T.E., 2009. Threshold-free cluster enhancement: addressing problems of smoothing, threshold dependence and localisation in cluster inference. NeuroImage 44, 83-98. doi:10.1016/j.neuroimage.2008.03.061

Speckmann, E.J., Altrup, U., 1993. Generation of cortical field potentials. Basic Mechanisms of the EEG 29-40.

Srinivasan, R., Winter, W.R., Ding, J., Nunez, P.L., 2007. EEG and MEG coherence: measures of functional connectivity at distinct spatial scales of neocortical dynamics. J. Neurosci. Methods 166, 41-52. doi:10.1016/j.jneumeth.2007.06.026

Steriade, M., 2005. Cellular Substrates of Brain Rhythms.

Steriade, M., McCormick, D.A., Sejnowski, T.J., 1993. Thalamocortical oscillations in the sleeping and aroused brain. Science 262, 679.

Tang, V., Michaelis, R., \& Kwan, P. (2014). Psychobehavioral therapy for epilepsy. Epilepsy \& Behavior : E\&B, 32, 147-155. doi:10.1016/j.yebeh.2013.12.004

Traub, R.D., Jefferys, J.G.R., Whittington, M.A., 1997. Simulation of gamma rhythms in networks of interneurons and pyramidal cells. Journal of computational neuroscience 4, 141-150.

van Albada, S.J., Kerr, C.C., Chiang, A., Rennie, C.J., Robinson, P.A., 2010. Neurophysiological changes with age probed by inverse modeling of EEG spectra. Clinical neurophysiology 121, 21-38.

Van Boxtel, A., 2001. Optimal signal bandwidth for the recording of surface EMG activity of facial, jaw, oral, and neck muscles. Psychophysiology 38, 22-34.

van Vugt, M.K., Jha, A.P., 2011. Investigating the impact of mindfulness meditation training on working memory: a mathematical modeling approach. Cogn Affect Behav Neurosci 11, 344-353. doi:10.3758/s13415-011-0048-8

Wallace, B.A., 2005. Balancing The Mind: A Tibetan Buddhist Approach To Refining 
Atention. Snow Lion Publications.

Wallace, B.A., 2006. The attention revolution: Unlocking the power of the focused mind. Wisdom Pubns.

Wallace, R.K., 1970. Physiological effects of transcendental meditation. Science 167, 1751.

Wilson, H.R., Cowan, J.D., 1972. Excitatory and inhibitory interactions in localized populations of model neurons. Biophysical journal 12, 1-24.

Wilson, H.R., Cowan, J.D., 1973. A mathematical theory of the functional dynamics of cortical and thalamic nervous tissue. Biol Cybern 13, 55-80.

Wilson, M., Bower, J.M., 1992. Cortical oscillations and temporal interactions in a computer simulation of piriform cortex. J Neurophysiol 67, 981.

Wright, J.J., Kydd, R.R., Sergejew, A.A., 1990. Autoregression models of EEG. Biol Cybern 62, 201-210.

Zhang, J.Z., others, 1988. EEG findings during special psychical state (Qi Gong state) by means of compressed spectral array and topographic mapping. Computers in Biology and Medicine 18, 455-463.

Zikopoulos, B., Barbas, H., 2006. Prefrontal projections to the thalamic reticular nucleus form a unique circuit for attentional mechanisms. Journal of Neuroscience 26, 73487361. doi:10.1523/JNEUROSCI.5511-05.2006

Zikopoulos, B., Barbas, H., 2007. Circuits for multisensory integration and attentional modulation through the prefrontal cortex and the thalamic reticular nucleus in primates. Reviews in the Neurosciences 18, 417.

Zylowska, L., Ackerman, D.L., Yang, M.H., Futrell, J.L., Horton, N.L., Hale, T.S., Pataki, C., Smalley, S.L., 2008. Mindfulness meditation training in adults and adolescents with ADHD: a feasibility study. J Atten Disord 11,737-746. doi:10.1177/1087054707308502

Zylowska, L., Smalley, S.L., Schwartz, J.M., 2009. Mindful Awareness and ADHD Springer. Clinical handbook of mindfulness. 
1276

1277

1278

1279

1280

1281

1282

1283

1284

1285

1286

1287

1288

1289

1290

1291

1292

1293

1294

1295

1296

1297

1298

1299

1300

1301

1302

1303

1304

1305

1306

1307

1308

1309

1310

1311

1312

1313

1314

1315

1316

1317

1318

1319

1320

1321

\section{Figure Legends:}

Figure 1: The cortico-cortical, cortico-thalamo-cortical, and intrathalamic parameters of the R-MFM. (a) A schematic of cortico-thalamic connections in the left hemisphere, via the reticular nucleus of the thalamus (TRN). (b) Basic neuronal physiology incorporated in the model, shown in a cortical neuron. Synaptic connections at the dendritic tree are shown emanating from pulse-rate fields $\phi_{\mathrm{b}}$, where, $\mathrm{b}=\mathrm{e}, \mathrm{i}, \mathrm{s}$ for cortical excitatory, inhibitory and subcortical connections, respectively. Somatic membrane potential is shown as $V_{a}$, where $a=e, i$ with resultant impulse firing rate $Q_{a}$ and the spread of action potentials as field $\phi_{\mathrm{a}}$, along the axons. (c) A schematic showing primary pathways between cortex, TRN, and thalamic secondary relay nuclei (SRN). Dashed lines represent inhibitory connections while solid lines represent excitatory ones. $\mathrm{G}_{\mathrm{ei}}$ depicts the projections between local excitatory and inhibitory neurons in the cortex, while $\mathrm{G}_{\mathrm{ee}}$ depicts similar projections between excitatory cortical neurons. These excitatory pyramidal neurons also project (through $\phi_{\mathrm{e}}$ ) to the thalamus, where signals may propagate (i) via TRN and then $S R N$ with gain $G_{\text {esre }}=G_{e s} G_{s r} G_{r e}$, or (ii) directly via SRN with gain $\mathrm{G}_{\text {ese }}=\mathrm{G}_{\mathrm{es}} \mathrm{G}_{\mathrm{se}}$. The $\mathrm{SRN}$ projects back (through $\phi_{\mathrm{s}}$ ) to the cortex, parametrized as the gain $G_{e s}$. Within the thalamus, the intrathalamic loop has the gain $G_{s r s}=G_{s r} G_{r s}$. Cortical activation (through sensory input) occurs via $\phi_{\mathrm{n}}$ and $\phi_{\mathrm{s}}$ with the gain $\mathrm{G}_{\mathrm{es}} \mathrm{G}_{\mathrm{sn}}$. The diagram is adapted from (Rowe et al., 2005). (d) Model parameters that were inspected using longitudinal analysis. Five model parameters related to the strength or efficacy of the corresponding loops are shown in the figure: cortico-cortical excitatory ( $\mathrm{G}_{\mathrm{ee}}$; in green), cortico-cortical inhibitory ( $\mathrm{G}_{\mathrm{ei}}$; in red), cortico-thalamo-cortical via TRN ( $\mathrm{G}_{\text {esre }}$; in blue), cortico-thalamo-cortical without TRN ( $\mathrm{G}_{\mathrm{ese}}$; in purple), and intrathalamic loop $\left(\mathrm{G}_{\mathrm{srs}}\right.$; in orange). Five other parameters (not shown in the figure) were also inspected longitudinally: cortical damping rate $\left(\gamma_{\mathrm{e}}\right)$, dendritic decay rate $(\alpha)$, conduction delay in the signal from cortex to thalamus and back $\left(\mathrm{t}_{0}\right)$, normalization parameter for the spectrum $\left(\mathrm{P}_{0}\right)$, and normalization parameter for the EMG component $(\mathrm{A})$.

Figure 2: Experimental design for longitudinal analysis of changes in model parameters due to training. After fitting the model for each assessment, the null hypotheses were tested for each group using the nonparametric cluster-based permutation analysis, followed by False Discovery Rate (FDR) correction for 30 nonparametric tests. The direction of effects, i.e. how parameter values change from T1 to T2 to T3, were examined with a Wilcoxon's test (with Bonferroni protection). Overall, this extension provides a statistical approach for longitudinal analysis of changes in the model parameters across all channels.

Figure 3: Measuring interactions in the modeled TRN layer. (a) Two units of the model and their interactions (dashed lines) in the TRN layer are shown. Ideally, parameter values for such interactions should be fitted along with all the other parameters of the model. However, given 73 units in total, this approach will lead to a combinatorial explosion of the fitting procedure. Thus, we developed a novel approach as described in (c). (b) The three layers of the model. Each column is modeled independently using the LEV approach. (c) The procedure formalizing within-TRN connectivity. After fitting the model to real EEG data, white noise was injected in the cortical cells and the resulting 
1322

1323

1324

1325

1326

1327

1328

1329

1330

1331

1332

1333

1334

1335

1336

1337

1338

1339

1340

1341

1342

1343

1344

1345

1346

1347

1348

1349

1350

1351

1352

1353

1354

1355

1356

1357

1358

1359

1360

1361

1362

1363

1364

1365

1366

1367

activity in the TRN cells was measured. Connectivity analysis in the TRN layer was then performed. (d) An example correlation matrix of injected white noise (no off-diagonal correlations present) and the resulting correlation matrix from TRN cells for a representative participant are shown. The diagonal elements in the TRN layer are set to zero for clarity. Instead of random ordering, an organized structure is evident in the correlation matrix of TRN cells, suggesting that even though these cells were modeled independently, connectivity information between them can still be discovered.

Figure 4: Results from model fitting of the EEG data for representative participants across scalp and groups. Real EEG spectrum is shown in solid black line and modeled EEG spectrum in dashed red line. Each column shows a different mid-line channel across the scalp (out of seventy-three channels, due to space limitations). There is close correspondence between the model spectra and experimental spectra across the scalp, participants, and groups.

Figure 5: Reduction in intrathalamic gain $\left(\mathrm{G}_{\mathrm{srs}}\right)$ during meditation. The color bars represent F-statistics. Using nonparametric cluster-based permutation approach, significant clusters were found in RG1 and RG2, whereas no clusters were found in CG. Clusters from RG1 and RG2 were used to extract data from CG participants, separately for each retreat, for post-hoc comparison and plotting of bar graphs. Overall, the reduction in intrathalamic gain during meditation was replicated across the retreats in similar spatial locations and no effect was found for the CG using either cluster. Error bars represent standard error of the mean.

Figure 6: Increase in corticothalamic delay $\left(\mathrm{t}_{0}\right)$ during meditation. The color bars represent F-statistics. No clusters were found in the CG. Clusters from RG1 and RG2 were used to extract data from CG participants, separately for each retreat, for post-hoc comparison and plotting of bar graphs. Overall, increased cortico-thalamic delay was found in parietal-occipital regions, in both retreat groups, at the middle and the end of the retreat. Error bars represent standard error of the mean.

Figure 7: Stability analysis during meditation. The bar chart depicts a significant drop in intra-thalamic stability parameter (z) in both retreat groups, suggesting increased stability as a result of training. No changes were found in the CG. Error bars represent standard error of the mean.

Figure 8: Connectivity analysis of the TRN layer. (a) Representative single participant posterior and anterior clusters, found after k-means clustering on the correlation matrix of TRN cells. (b) Average topographical maps (over all participants) for each assessment and group are shown during the meditation state. For each participant, each channel was given a value of +1 (when in the anterior cluster) or -1 (when in the posterior cluster). Overall, clear separation between the anterior and poster regions of the modeled TRN was found. (c) Between clusters connectivity analysis revealed increased connectivity between anterior and posterior clusters associated with meditation training. Error bars represent standard error of the mean. 
Tables:

Table 1: Initial values and physiologically restricted limits of model parameters in the current study as prescribed by (Rowe et al., 2004a). The ten model parameters that were varied during data fitting are indicated with an asterisk $(*)$ next to their initial values. The limits, fixed values, and initial values are in line with the independent sources and physiological measures (Robinson et al., 1997; Rowe et al., 2004b; Shwedyk et al., 1977; Van Boxtel, 2001).

\begin{tabular}{|c|c|c|c|c|}
\hline Model & Parameter & Description & $\begin{array}{l}\text { Physiological } \\
\text { Limits }\end{array}$ & Initial Value \\
\hline \multirow[t]{13}{*}{$\begin{array}{l}\text { EEG } \\
\text { parameters }\end{array}$} & $\gamma_{\mathrm{e}}$ & $\begin{array}{l}\text { Cortical damping parameter to } \\
\text { incorporate reduction in signal with } \\
\text { increasing axonal distance }\end{array}$ & {$[40,400]$} & $\begin{array}{l}130 \text { per } \\
\text { second* }\end{array}$ \\
\hline & $\alpha$ & $\begin{array}{l}\text { Dendritic decay rate parameter to } \\
\text { incorporate temporal spread and } \\
\text { conduction delay in the dendritic tree }\end{array}$ & {$[10,200]$} & $\begin{array}{l}75 \text { per } \\
\text { second* }\end{array}$ \\
\hline & $\beta$ & $\begin{array}{l}\text { Dendritic rise rate parameter to } \\
\text { incorporate temporal spread and } \\
\text { conduction delay in the dendritic tree }\end{array}$ & Depends on $\alpha$ & $\begin{array}{l}3.8 \times \alpha \text { per } \\
\text { second }\end{array}$ \\
\hline & $\mathrm{t}_{0}$ & $\begin{array}{l}\text { Conduction delay parameter to } \\
\text { incorporate temporal delay in signal } \\
\text { transmission from cortex to thalamic } \\
\text { nuclei and back }\end{array}$ & {$[0.06,0.13]$} & $\begin{array}{l}0.084 \\
\text { seconds* }\end{array}$ \\
\hline & $\mathrm{G}_{\mathrm{ee}}$ & $\begin{array}{l}\text { Excitatory gain parameter to incorporate } \\
\text { connection strength from pyramidal cells }\end{array}$ & {$[0,50]$} & $5.4^{*}$ \\
\hline & $\mathrm{G}_{\mathrm{ei}}$ & $\begin{array}{l}\text { Local intra-cortical inhibitory gain } \\
\text { parameter to incorporate connection } \\
\text { strength from stellate cells }\end{array}$ & {$[-35,-1]$} & $-7 *$ \\
\hline & $\mathrm{G}_{\mathrm{ese}}$ & $\begin{array}{l}\text { First corticothalamocortical gain } \\
\text { parameter to incorporate combined } \\
\text { strength of connections from cortex to } \\
\text { secondary relay nuclei and back to } \\
\text { cortex }\end{array}$ & {$[0,50]$} & $5.6^{*}$ \\
\hline & $\mathrm{G}_{\text {esre }}$ & $\begin{array}{l}\text { Second corticothalamocortical gain } \\
\text { parameter to incorporate combined } \\
\text { strength of connections from cortex to } \\
\text { reticular nucleus to secondary relay } \\
\text { nuclei of thalamus and back to cortex }\end{array}$ & {$[-30,0]$} & $-2.8^{*}$ \\
\hline & $\mathrm{G}_{\mathrm{srs}}$ & $\begin{array}{l}\text { Intrathalamic gain parameter to } \\
\text { incorporate connection strength within } \\
\text { two thalamic nuclei (TRN and SRN) }\end{array}$ & {$[-15,0.5]$} & $-0.6^{*}$ \\
\hline & $\mathrm{k}_{0} \mathrm{r}_{\mathrm{e}}$ & Volume conduction filter parameter & Fixed & 3.0 a.u. \\
\hline & $l_{x}, l_{y}$ & Linear dimensions of cortex & Fixed & 0.5 meters \\
\hline & $\mathrm{r}_{\mathrm{e}}$ & Characteristic pyramidal axon length & Fixed & 0.08 meters \\
\hline & $\mathrm{P}_{0}$ & Overall power normalization & $\begin{array}{l}\text { Estimated from } \\
\text { the data }\end{array}$ & $\begin{array}{l}\text { Estimated } \\
\text { from the data* }\end{array}$ \\
\hline \multirow{3}{*}{$\begin{array}{l}\text { EMG } \\
\text { parameters }\end{array}$} & $\mathrm{A}$ & Power normalization & {$[0,99]$} & $0.5 \mu \mathrm{V}^{2} / \mathrm{Hz}^{*}$ \\
\hline & $\mathrm{f}_{\mathrm{pk}}$ & Peak frequency of the EMG spectrum & Fixed & $40 \mathrm{~Hz}$ \\
\hline & $\delta$ & Asymptotic slope parameter & Fixed & 2 a.u. \\
\hline
\end{tabular}


Table 2: Goodness of fit results based on R-square values. The results are averaged over all channels and participants for each assessment and group, after fitting to the real EEG spectrum. Overall, the model accounted for more than $98 \%$ variance in all the cases. consistently across the scalp and the participants.

\begin{tabular}{ccccccc}
\hline & \multicolumn{2}{c}{$\mathbf{R G 1}$} & \multicolumn{2}{c}{$\mathbf{C G}$} & \multicolumn{2}{c}{$\mathbf{R G 2}$} \\
& $\boldsymbol{\mu} \mathbf{R}_{\text {square }}$ & $\boldsymbol{\sigma} \mathbf{R}_{\text {square }}$ & $\boldsymbol{\mu} \mathbf{R}_{\text {square }}$ & $\boldsymbol{\sigma R}_{\text {square }}$ & $\boldsymbol{\mu} \mathbf{R}_{\text {square }}$ & $\boldsymbol{\sigma R}_{\text {square }}$ \\
\hline $\mathrm{T} 1$ & 0.985 & 0.0055 & 0.984 & 0.0053 & 0.983 & 0.0063 \\
$\mathrm{~T} 2$ & 0.983 & 0.0076 & 0.985 & 0.0057 & 0.984 & 0.0057 \\
$\mathrm{~T} 3$ & 0.984 & 0.0061 & 0.985 & 0.0063 & 0.983 & 0.0069 \\
\hline
\end{tabular}

1386

1387

1388

1389

1390

1391

1392

Table 3: Mean values of fitted model parameters for each set of participants (RG1, CG, and RG2) and assessments (T1, T2, and T3). The values are averaged over all channels and participants after fitting to the real EEG spectrum collected during meditation state. As evident, the final parameter values are within the physiologically plausible range (refer to Table 1).

\begin{tabular}{lccccccccccc}
\hline & & $\boldsymbol{\alpha}$ & $\mathbf{t}_{\mathbf{0}}$ & $\mathbf{G}_{\text {srs }}$ & $\boldsymbol{\gamma}_{\mathbf{e}}$ & $\mathbf{G}_{\text {ee }}$ & $\mathbf{G}_{\text {ei }}$ & $\mathbf{G}_{\text {esre }}$ & $\mathbf{G}_{\text {ese }}$ & $\mathbf{p}_{\mathbf{0}}$ & \multicolumn{1}{c}{$\mathbf{A}$} \\
\hline RG1 & $\mathrm{T} 1$ & 113.7 & 0.0889 & -0.8977 & 282.7 & 24.5 & -29.93 & -4.99 & 15.12 & 0.1652 & 7.8 \\
& $\mathrm{~T} 2$ & 116.9 & 0.0865 & -0.8027 & 287.1 & 24.7 & -30.34 & -3.73 & 14.48 & 0.1468 & 6.8 \\
& $\mathrm{~T} 3$ & 113.8 & 0.0860 & -0.8471 & 287.9 & 24.0 & -30.10 & -3.94 & 15.54 & 0.1694 & 7.2 \\
$\mathrm{CG}$ & $\mathrm{T} 1$ & 112.0 & 0.0863 & -0.9474 & 275.6 & 24.0 & -29.89 & -3.86 & 16.31 & 0.2702 & 8.1 \\
& $\mathrm{~T} 2$ & 113.2 & 0.0850 & -0.9288 & 281.8 & 25.0 & -30.75 & -3.64 & 15.04 & 0.2117 & 7.9 \\
& $\mathrm{~T} 3$ & 111.2 & 0.0850 & -0.9295 & 273.6 & 23.9 & -29.87 & -3.81 & 16.38 & 0.2508 & 7.6 \\
$\mathrm{R} 62$ & $\mathrm{~T} 1$ & 111.6 & 0.0913 & -0.8419 & 296.1 & 25.5 & -30.68 & -4.95 & 15.50 & 0.2244 & 9.4 \\
& $\mathrm{~T} 2$ & 112.4 & 0.0897 & -0.8046 & 293.9 & 26.1 & -31.16 & -4.35 & 15.02 & 0.2051 & 8.9 \\
& $\mathrm{~T} 3$ & 114.6 & 0.0896 & -0.8030 & 287.8 & 25.8 & -30.26 & -4.72 & 14.83 & 0.1937 & 8.9 \\
\hline
\end{tabular}

Table 4: Average standard deviation values of fitted model parameters for each set of participants (RG1, CG, and RG2) and assessments (T1, T2, and T3).

\begin{tabular}{llllllllllll}
\hline & & $\boldsymbol{\alpha}$ & $\mathbf{t}_{\mathbf{0}}$ & $\mathbf{G}_{\text {srs }}$ & $\gamma_{\mathbf{e}}$ & $\mathbf{G}_{\text {ee }}$ & $\mathbf{G}_{\text {ei }}$ & $\mathbf{G}_{\text {esre }}$ & $\mathbf{G}_{\text {ese }}$ & $\mathbf{p}_{\mathbf{0}}$ & $\mathbf{A}$ \\
\hline RG1 & $\mathrm{T} 1$ & 13.53 & 0.0047 & 0.4006 & 57.31 & 3.09 & 2.07 & 2.53 & 4.14 & 0.1384 & 4.09 \\
& $\mathrm{~T} 2$ & 13.9 & 0.0056 & 0.2812 & 49.51 & 3.17 & 2.4 & 2.72 & 4.92 & 0.1245 & 3.69 \\
& $\mathrm{~T} 3$ & 13.03 & 0.006 & 0.3318 & 52.82 & 4.04 & 2.32 & 3.01 & 4.92 & 0.1552 & 4.37 \\
$\mathrm{CG}$ & $\mathrm{T} 1$ & 11.21 & 0.0066 & 0.4187 & 55.6 & 2.77 & 2.05 & 2.01 & 4.62 & 0.191 & 2.91 \\
& $\mathrm{~T} 2$ & 11.27 & 0.0072 & 0.4193 & 62.91 & 3.25 & 2.55 & 2.5 & 6.22 & 0.1578 & 2.89 \\
& $\mathrm{~T} 3$ & 11.12 & 0.0064 & 0.4239 & 60.13 & 3.61 & 1.99 & 2.22 & 6.31 & 0.1849 & 2.64 \\
$\mathrm{R}$ R2 & $\mathrm{T} 1$ & 10.9 & 0.0059 & 0.3918 & 65.96 & 3.16 & 2.12 & 2.84 & 5.14 & 0.1696 & 4.13 \\
& $\mathrm{~T} 2$ & 9.44 & 0.007 & 0.3619 & 66.38 & 3.54 & 2.9 & 2.71 & 5.39 & 0.1566 & 3.71 \\
& $\mathrm{~T} 3$ & 9.94 & 0.0077 & 0.3691 & 64.37 & 3.63 & 2.89 & 2.63 & 4.18 & 0.1687 & 3.81 \\
\hline
\end{tabular}


Figure 1

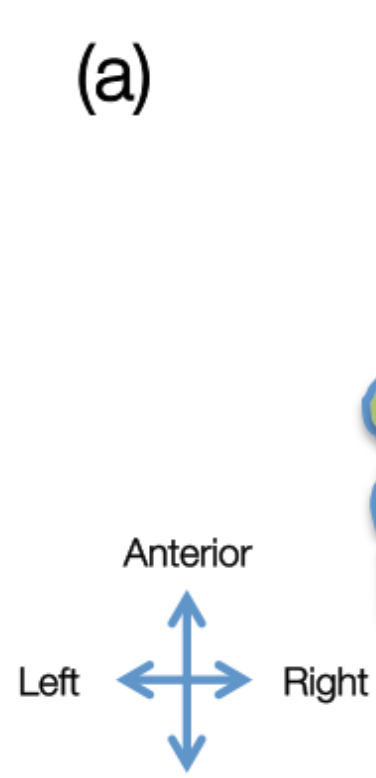

Posterior

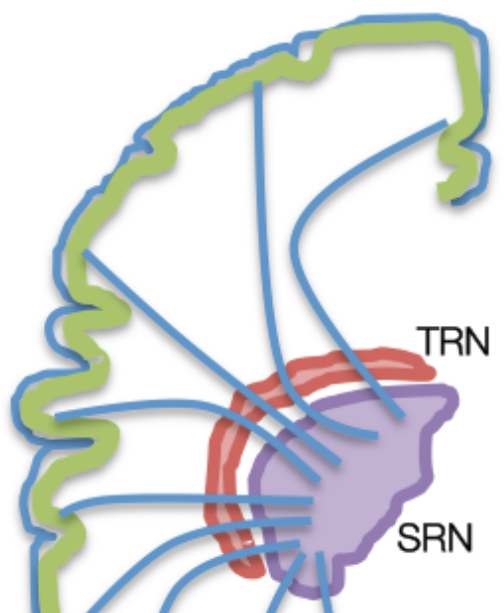

(c)

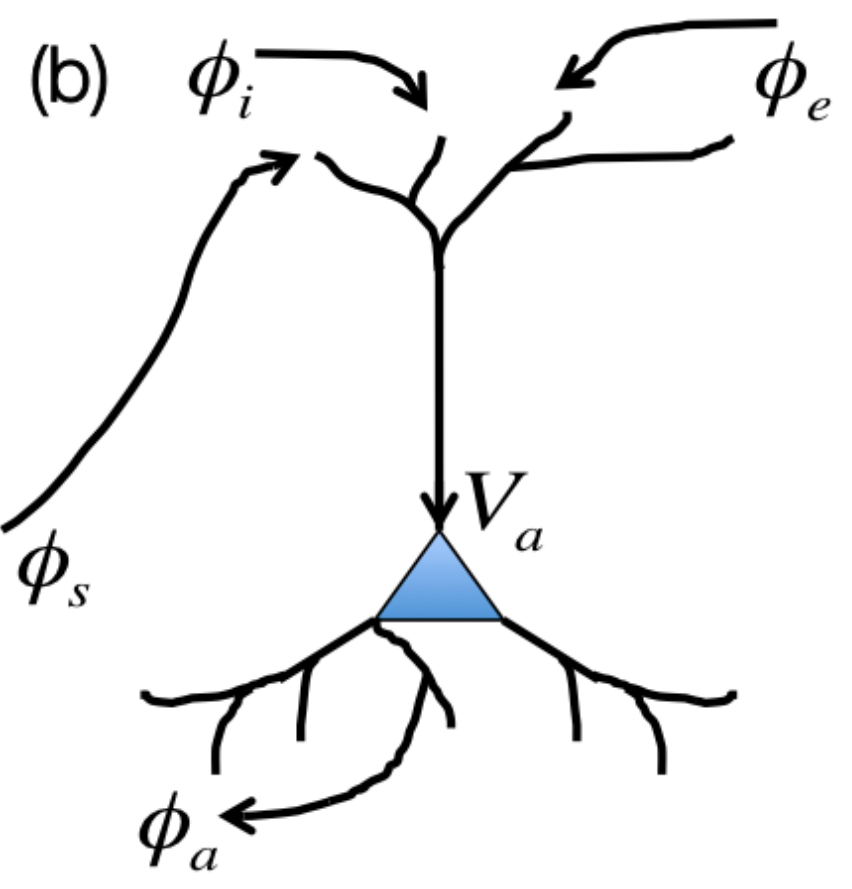

(d)

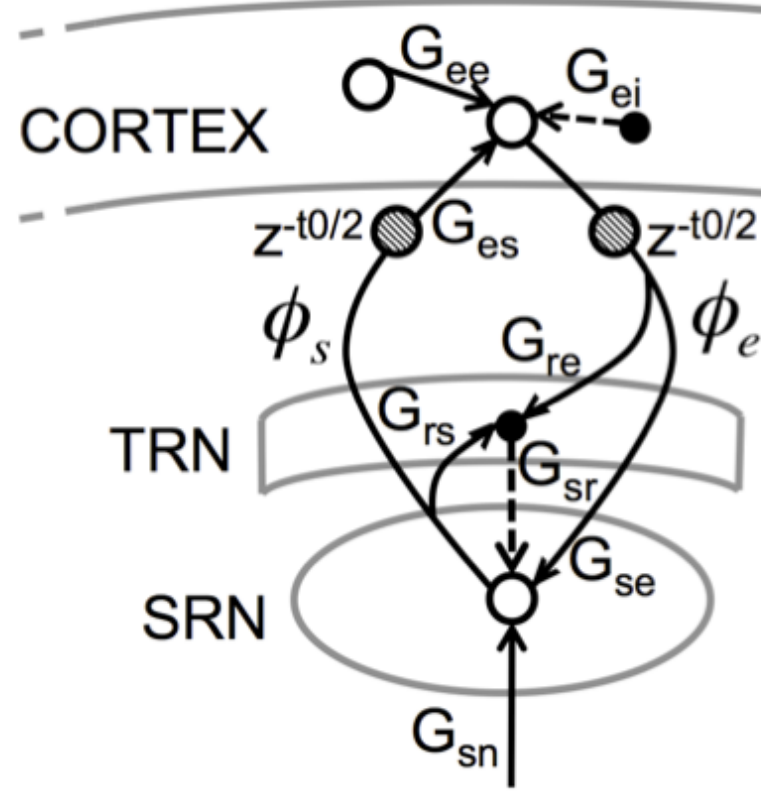

$\phi_{n}$

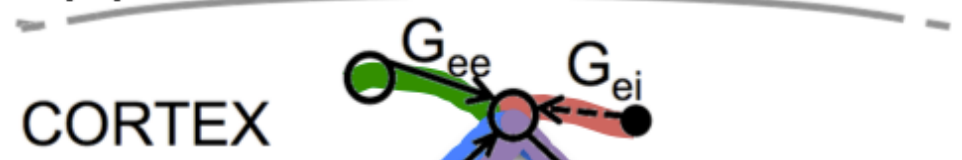

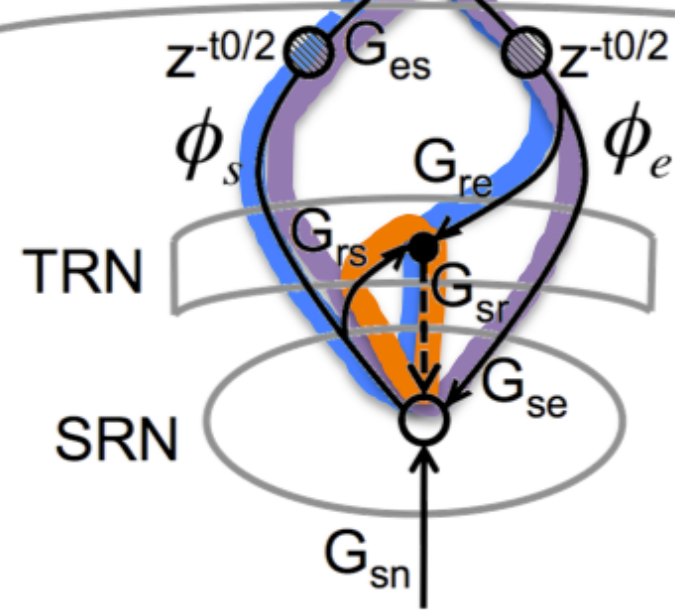

$\phi_{n}$ 
Figure 2

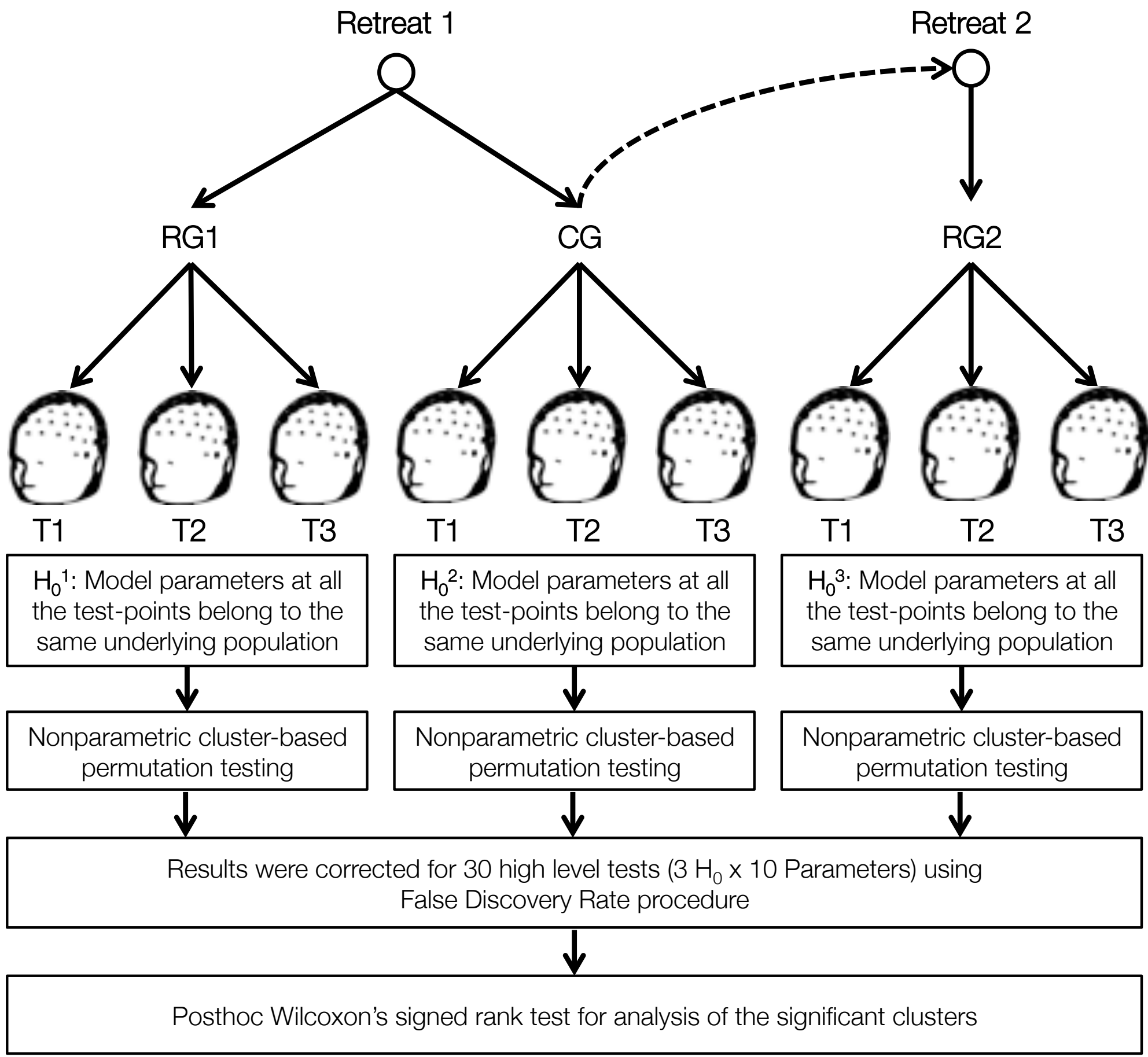


Figure 3

(a)

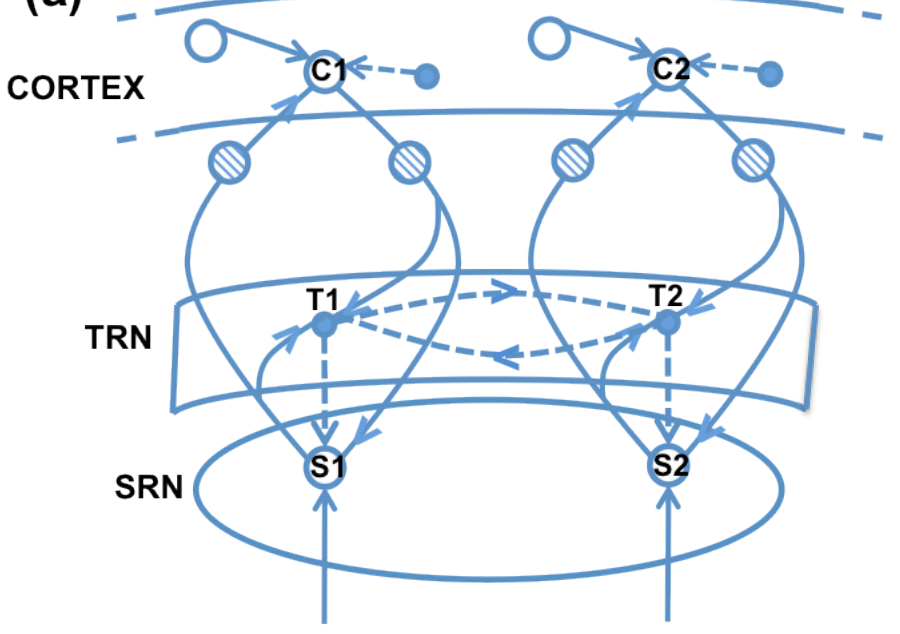

(c)

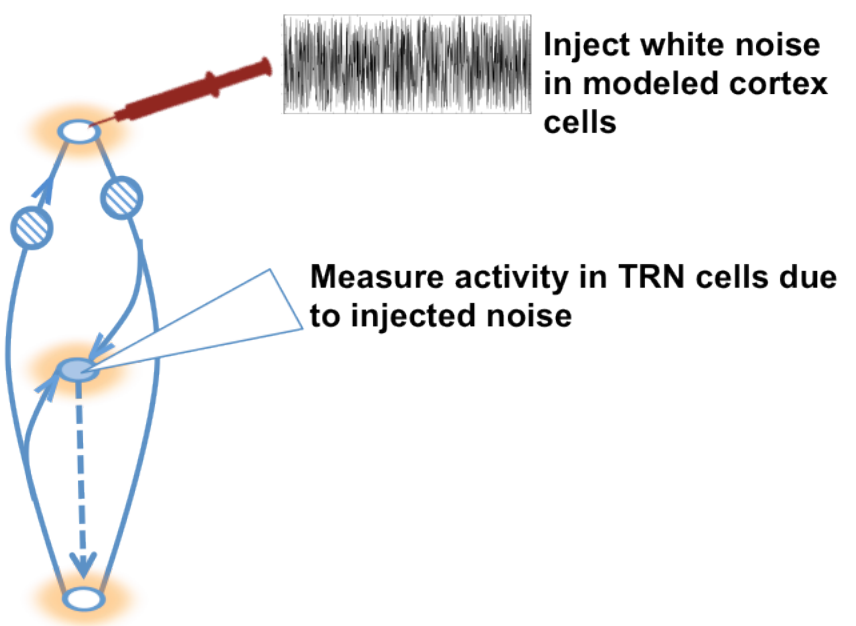

(b)

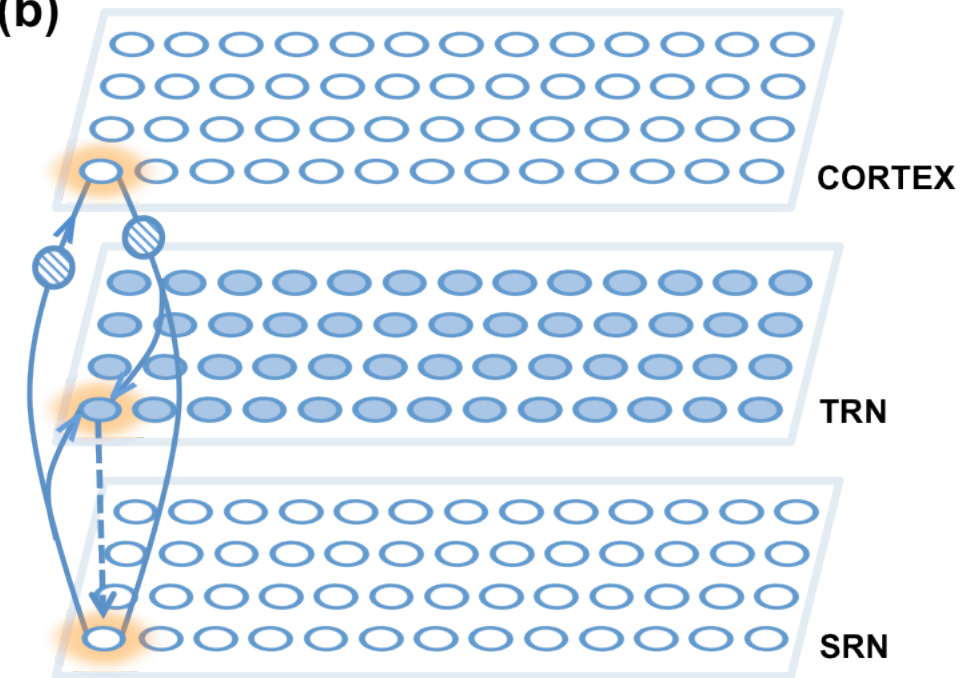

(d)

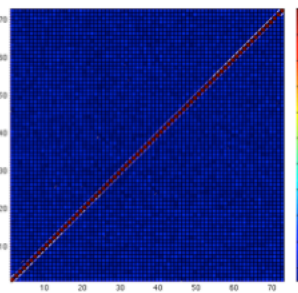

- Correlation matrix from injected ' noise

Clustered correlation matrix from measured signal in TRN

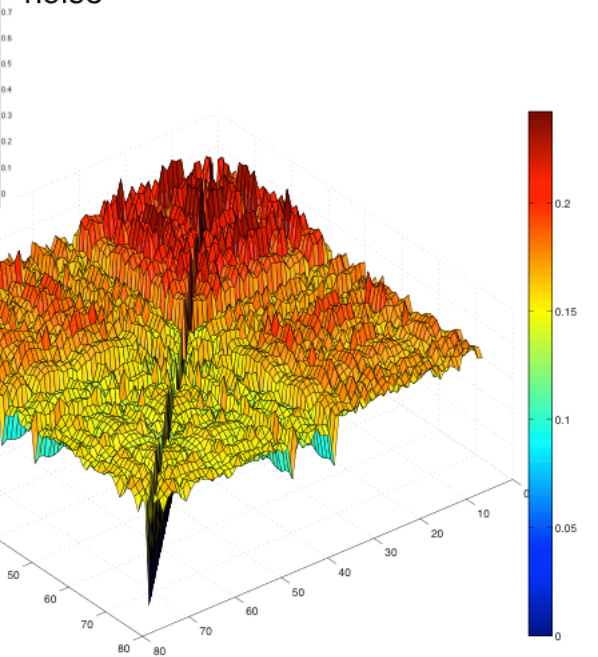


(A) RG1

Anterior Frontal (AFz)

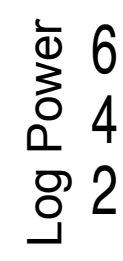

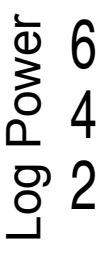

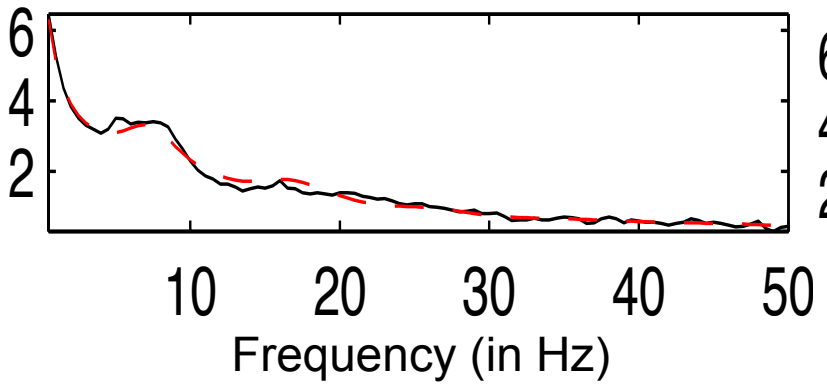

Central Parietal $(\mathrm{Cz})$

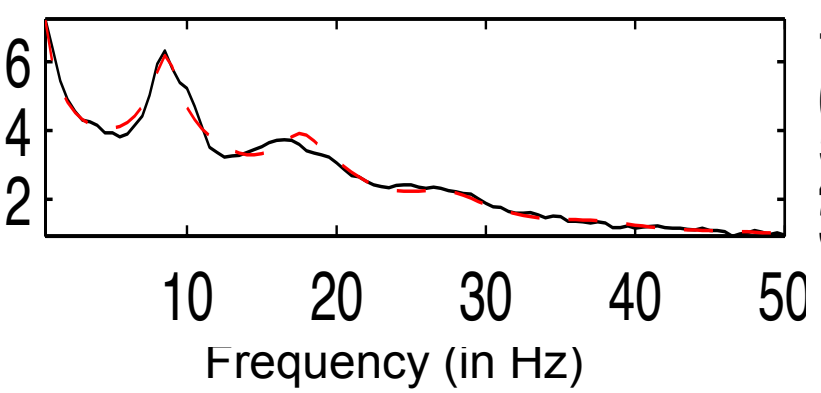

Occipital (Oz)

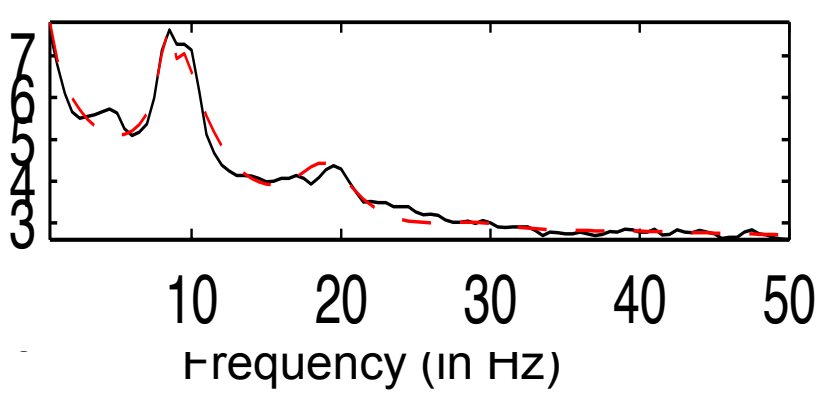

(B) CG

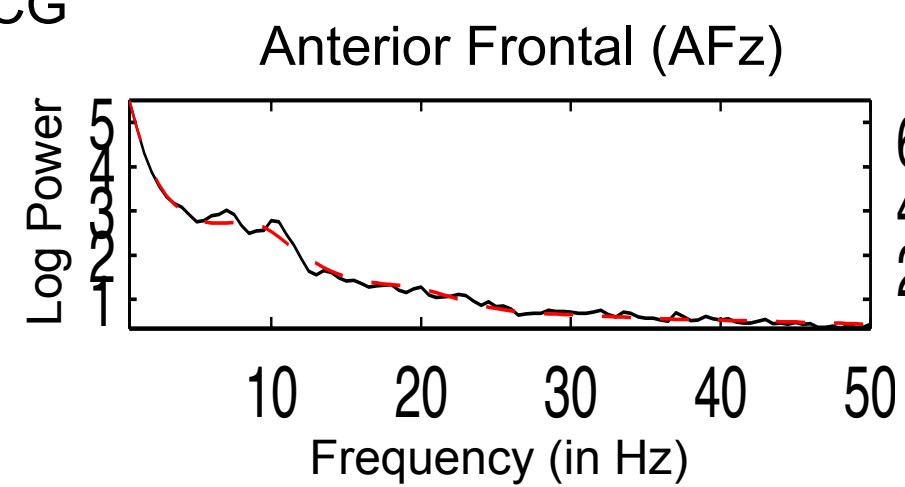

Central Parietal $(\mathrm{Cz})$

Occipital $(\mathrm{Oz})$
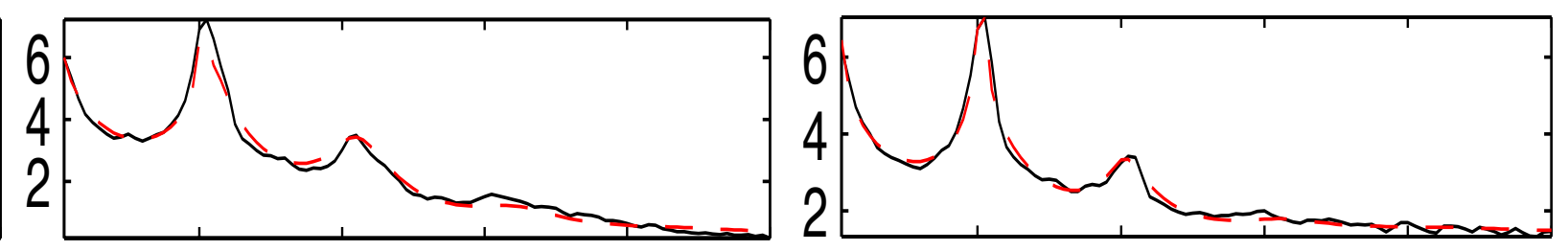

$\begin{array}{lllll}10 & 20 & 30 & 40 & 50\end{array}$ Frequency (in $\mathrm{Hz}$ )

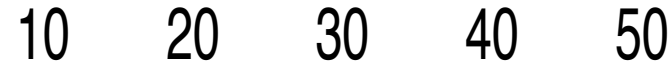

Frequency (in $\mathrm{Hz}$ )

(C) RG2 Anterior Frontal (AFz)

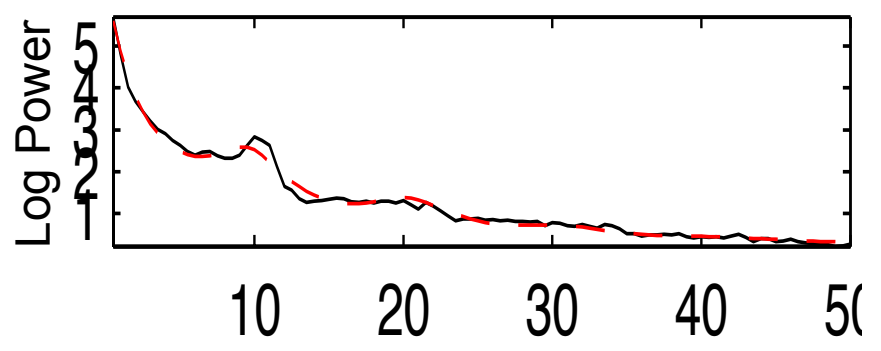

Frequency (in $\mathrm{Hz}$ )

Central Parietal $(\mathrm{Cz})$

Occipital (Oz)

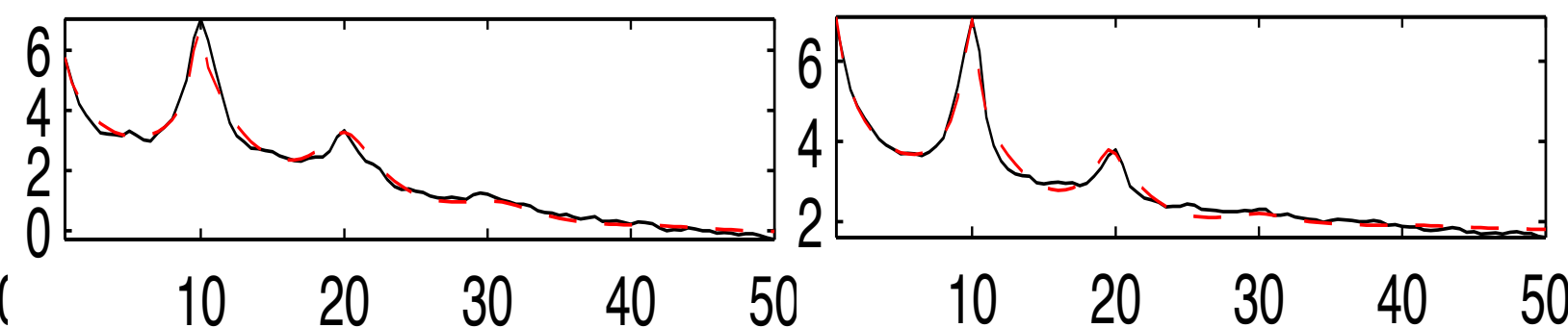

Frequency (in $\mathrm{Hz}$ )

Frequency (in $\mathrm{Hz}$ ) 
Figure 5

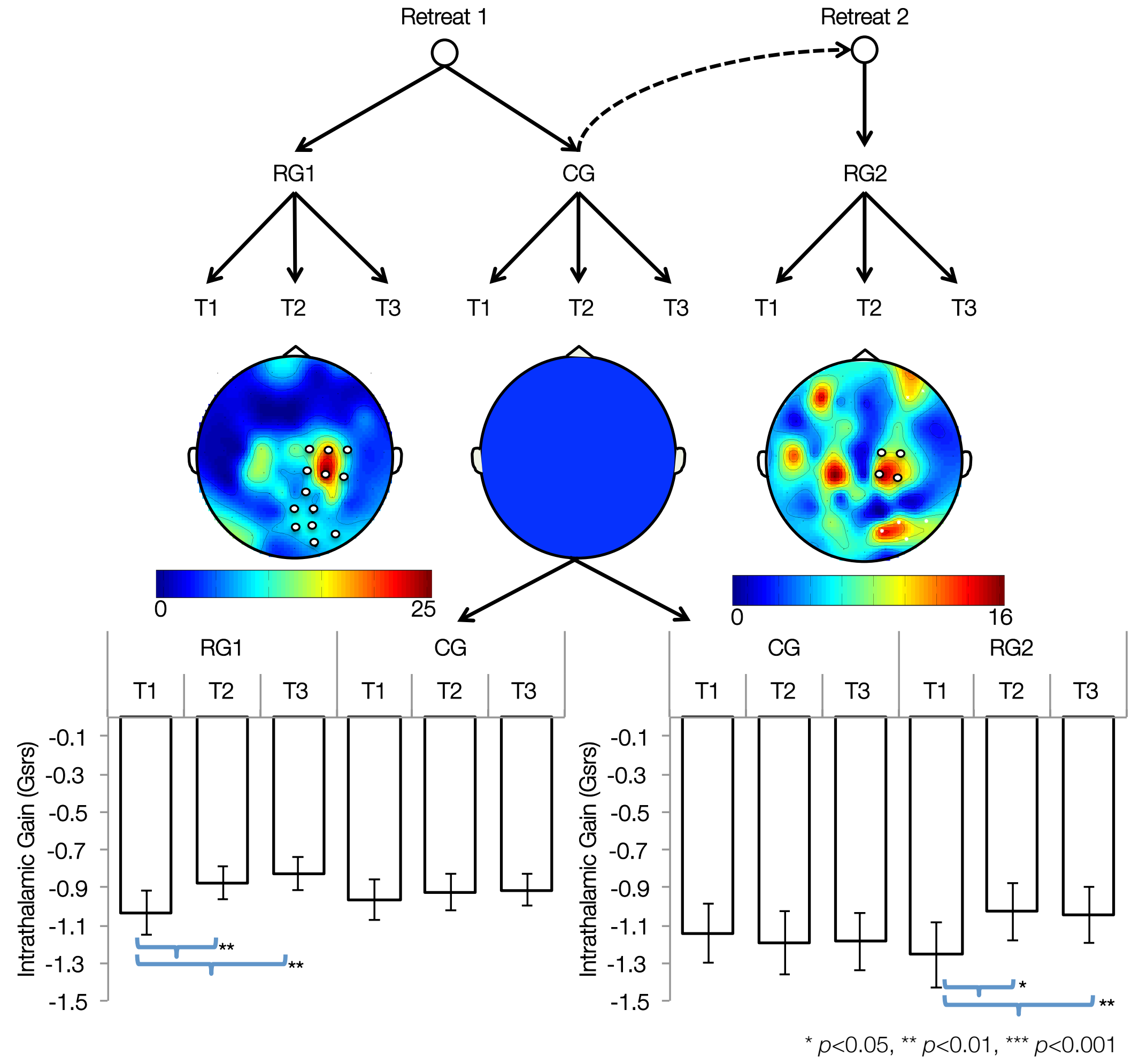


Figure 6

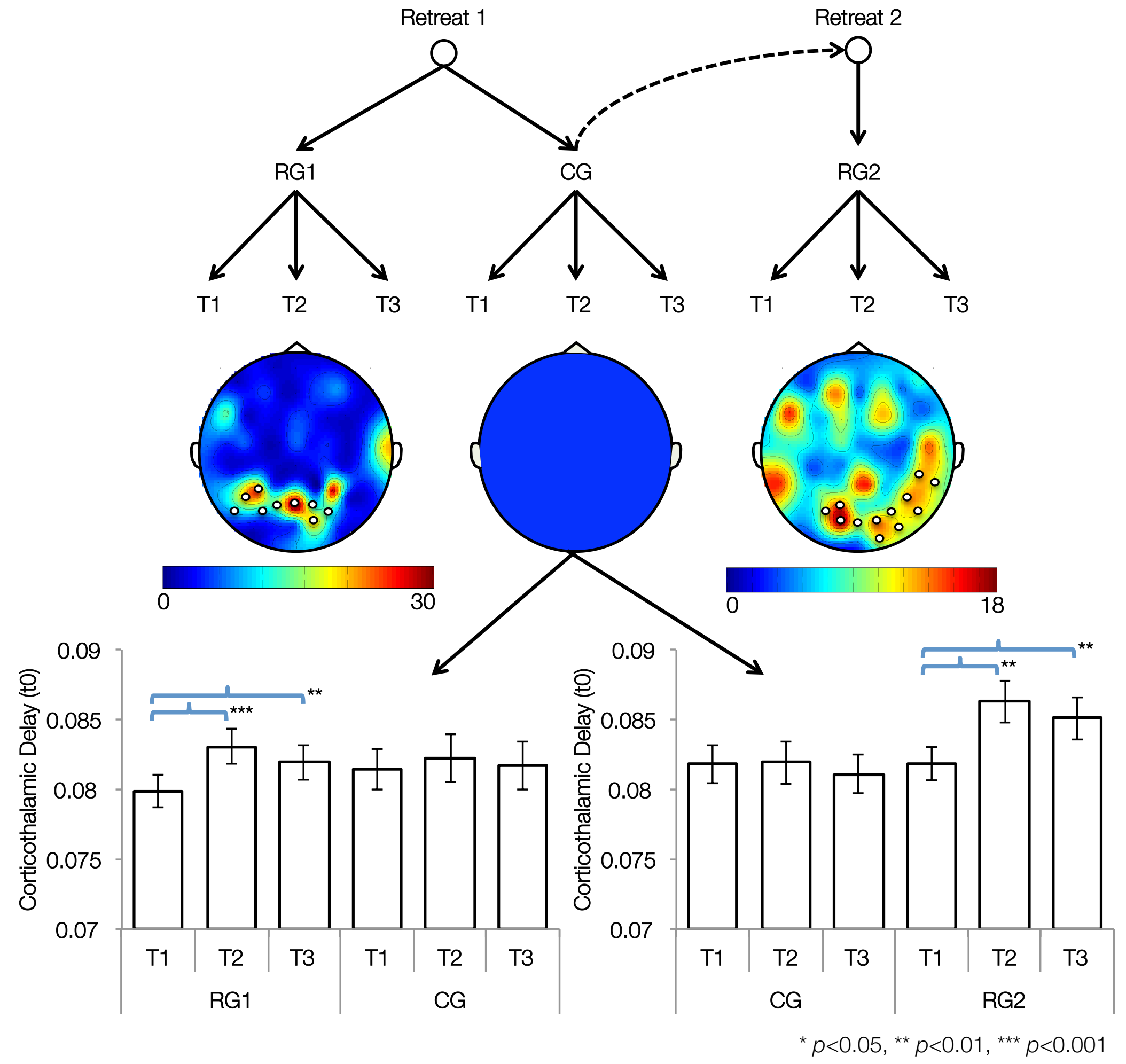


Figure 7
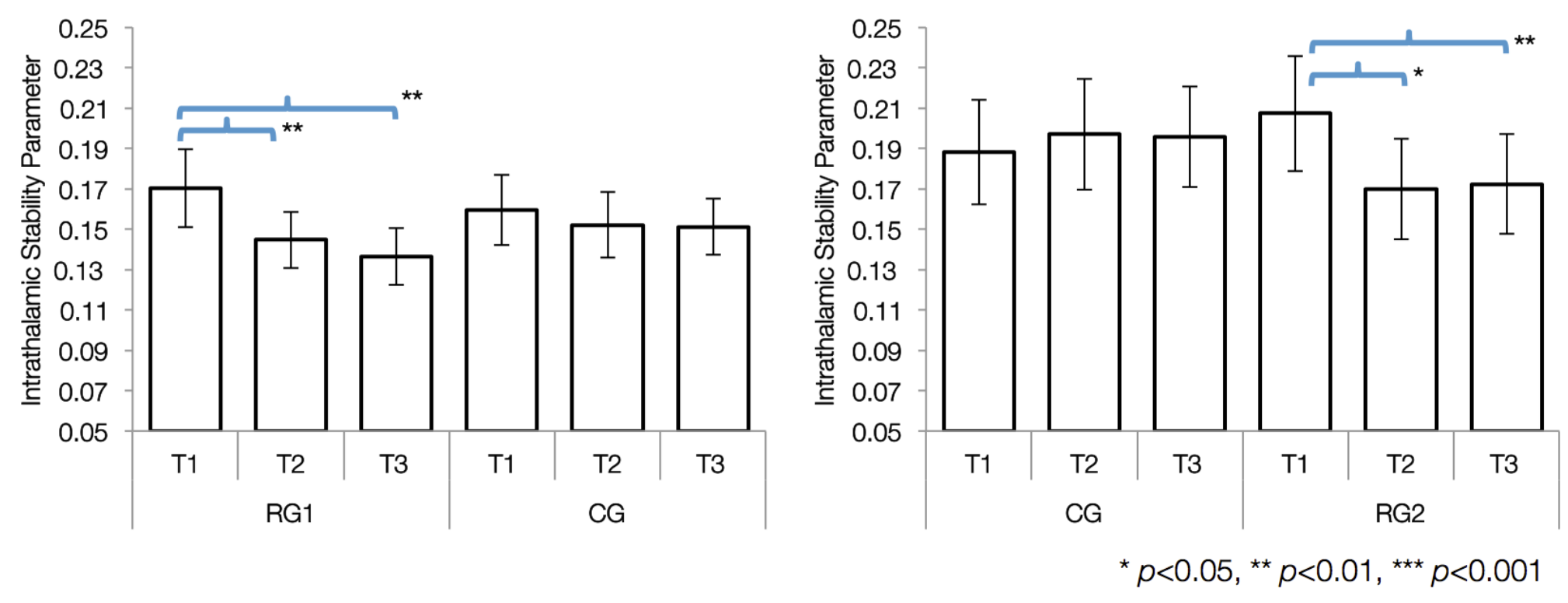
Figure 8

(a)

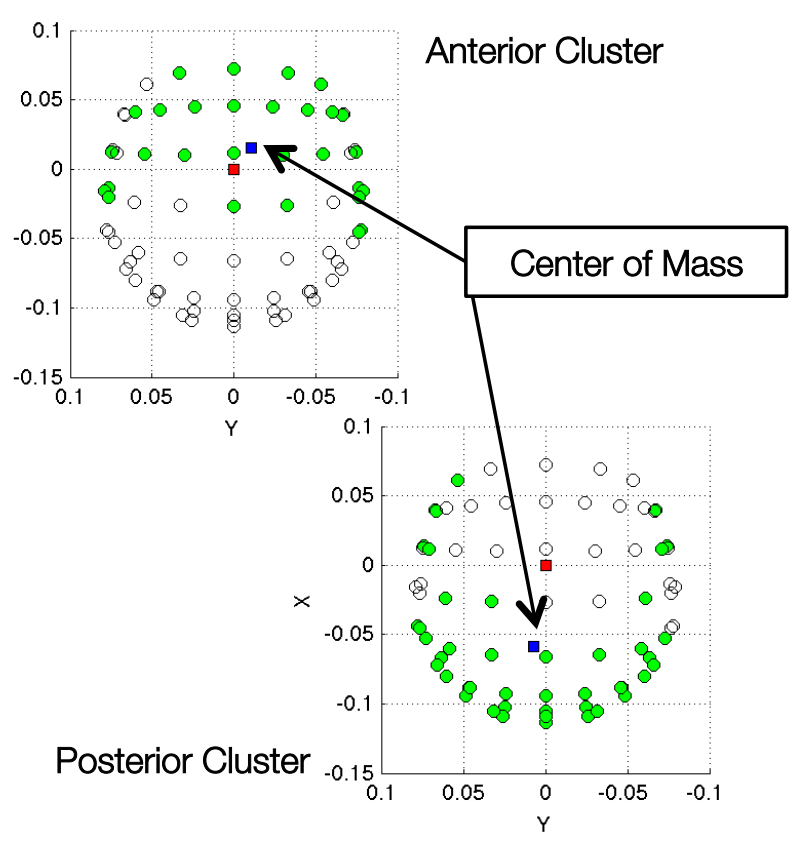

(b)

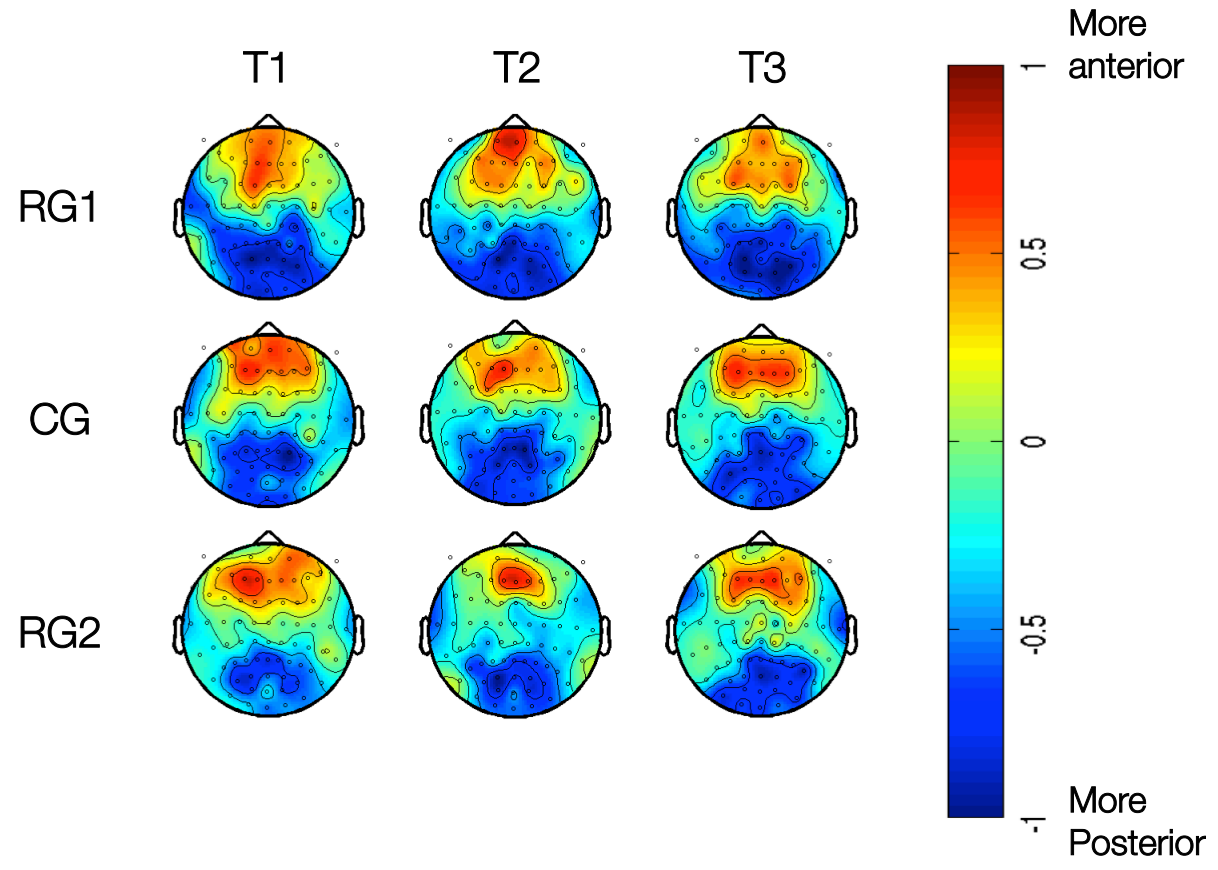

(c)

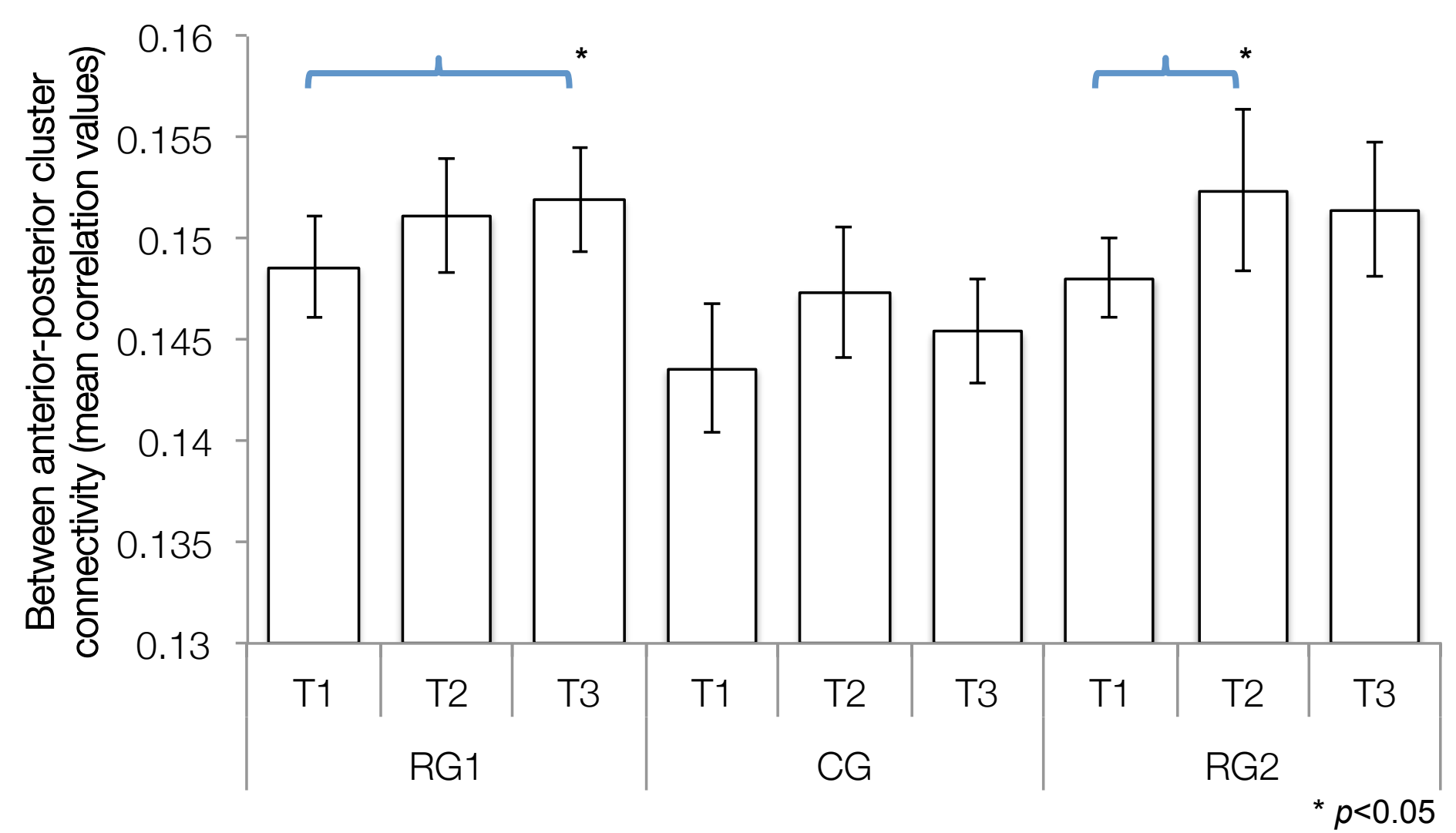

